\title{
FALDI-based decomposition of an atomic interaction line leads to 3D representation of the multicentre nature of interactions
}

\author{
Jurgens H. de Lange, Daniel M. E. van Niekerk and Ignacy Cukrowski
}

Correspondence to: Ignacy Cukrowski (E-mail: ignacy.cukrowski@up.ac.za)

Department of Chemistry, Faculty of Natural and Agricultural Sciences, University of Pretoria, Lynnwood Road, Hatfield, Pretoria 0002, South Africa

\begin{abstract}
Atomic interaction lines (AILs) and the QTAIM's molecular graphs provide a predominantly two-centre viewpoint of interatomic interactions. While such a bicentric interpretation is sufficient for most covalent bonds, it fails to adequately describe both formal multicentre bonds as well as many non-covalent interactions with some multicentre character. We present an extension to our Fragment, Atomic, Localized, Delocalized and Interatomic (FALDI) electron density (ED) decomposition scheme, with which we can measure how any atom-pair's delocalized density concentrates, depletes or reduces the electron density in the vicinity of a bond critical point. We apply our method on five classical bonds/interactions, ranging from formal either two- or three-centre bonds, a non-covalent interaction (an intramolecular hydrogen bond) to organometallic bonds with partial multicentre character. By use of 3D representation of specific atom-pairs contributions to the delocalized density we (i) fully recover previous notion of multicentre bonding in diborane and predominant bicentric character of a single covalent $\mathrm{C}-\mathrm{C}$ bond, (ii) reveal a multicentre character of an intramolecular H-bond and (iii) illustrate, relative to a Schrock carbene, a larger degree of multicentre $\mathrm{M}-\mathrm{C}$ interaction in a Fischer carbene (due to a presence of a heteroatom), whilst revealing the holistic nature of AILs from multicentre ED decomposition.
\end{abstract}

Keywords: Atomic Interaction Line, Electron Density Decomposition, FALDI, Multicentre Interaction, Computational Chemistry 


\section{Introduction}

The concept of multicentre bonding has been known to chemists for almost 75 years, yet most physical properties of a bond (such as bond lengths, dissociation and interaction energies or even degrees of atomic orbital overlap) are reported and interpreted in terms of a two-centre interaction. Multicentre bonding is "paradoxically counter intuitive in chemistry" because of chemists' lingering tradition of representing a bond as a line, to paraphrase Silvi. ${ }^{[1]}$ Despite such conceptual difficulties, a large number of interactions has been formally classified (by a multitude of methods) as multicentre bonds. For instance, the remarkable differences observed between the structures of $\mathrm{B}_{2} \mathrm{H}_{6}$ and $\mathrm{C}_{2} \mathrm{H}_{6}$ led to the discovery of a large number of diborane structures with multicentre bonds. ${ }^{[2-6]}$ Moreover, multiple ligands which can bridge metal-metal bonds - such as chlorine atoms or carbonyl ligands - have been discovered and are well-known examples of formal $\mathrm{M} \cdots \mathrm{X} \cdots \mathrm{M}$ multicentre bonds. ${ }^{[7-11]}$ Relatively recently, unusually long, multicentre bonds between dimers of tetracyanoethylene have attracted a large amount of interest, ${ }^{[12-14]}$ as well as 4-centre-2-electron bonding. ${ }^{[15]}$ Even metallic bonding, consisting of a 'sea of delocalized electrons', has been classified as multicentre bonding. ${ }^{[16]}$

A great number of two-centre bonds with known partial multicentre character exist, however, which are often overlooked. Hydrogen bonding is generally accepted to be an interaction caused by the combined effect of at least 3 atoms: an H-bond donor atom $(\mathrm{X})$, a hydrogen atom $(\mathrm{H})$ and an $\mathrm{H}$-bond acceptor atom $(\mathrm{Y})$. IUPAC suggests that the backbone atom bonded to $\mathrm{Y}$, known as atom $\mathrm{Z}$, could also be included in the definition in specific cases, ${ }^{[17,18]}$ and we recently provided evidence ${ }^{[19]}$ of the considerable role that the atom bonded to $\mathrm{X}$ (atom $\mathrm{W}$ ) plays in the formation of an intramolecular $\mathrm{H}$-bond in $\beta$-alanine. Clearly, an $\mathrm{H}$-bond involves contributions from more than only atoms $\mathrm{H}$ and $\mathrm{Y}$ as a conceptual line drawn between only these two atoms suggests. The nature and strength of many reported non-covalent interactions are greatly affected by their local environment and neighbouring atoms, ${ }^{[20]}$ potentially an indication of some degree of multicentre character (including an agostic bond in a ruthenium complex, which has been formally characterized as a multicentre bond $\left.{ }^{[1]}\right)$. Aromatic bonds in electron delocalized molecular systems are examples of partially multicentre bonding, as are any interactions involving an entire $\pi$-ring consisting of overlapping $p$-orbitals. Finally, the important influence of heteroatoms on organometallic $\mathrm{C}=\mathrm{M}$ carbene bonds ${ }^{[21]}$ is a clear indication of multicentre character on what is normally interpreted as a diatomic 'double' bond. For instance, the presence of a 
heteroatom in Fischer carbene complexes typically enhances $\pi$-backbonding from the metal atom, as well as $\sigma$-donation from the ligand in comparison to most Schrock carbenes.

The Quantum Theory of Atoms in Molecules (QTAIM) ${ }^{[22]}$ is a widely used tool in multiple applications of computational chemistry. QTAIM's molecular graphs present a topologically condensed view of the electron density (ED) as a series of critical points, where nuclear critical points $(+3,-3)$ can be connected to bond critical points, BCPs $(+3,-1)$ by atomic interaction lines (AILs, also known as bond paths). The discovery that AILs are found concomitant with every generally accepted chemical bond is a remarkable achievement of modern chemistry, despite the somewhat controversial occurrences of AILs being found where no chemical bond is expected. ${ }^{[23-26]}$ However, the connectivity provided by a molecular graph is strictly a two-centre viewpoint. An AIL is present between atoms $\mathrm{H}$ and $\mathrm{Y}$ in a typical H-bond, for instance, lending a two-centre impression of the bond rather than the multicentre interpretation as suggested by IUPAC. In electron delocalized systems, such as benzene, a two-centre interpretation becomes problematic and raises the question: to what extent does an AIL between two neighbouring carbon atoms in benzene represent a diatomic bonding interaction and/or the degree of aromatic delocalization of electrons from other atomic centres?

The extent to which a chemical interaction is multicentre seems to be a surprisingly difficult question to answer. Silvi suggested a density-based approach using the Electron Localization Function which is able to differentiate multicentre bonding (such as in $\mathrm{B}_{2} \mathrm{H}_{6}$ ) from two-centre bonding (such as in $\mathrm{C}_{2} \mathrm{H}_{6}$ ). ${ }^{[1]}$ Silvi's approach, however, produces very strict categorization (i.e. an interaction is strictly either two- or three-centre) and does not provide any insight regarding partial multicentre character (such as in the aforementioned carboncarbon interaction in benzene). Ponec et al..$^{[9,27-29]}$ have studied multicentre bonding within the QTAIM framework extensively, particularly with the use of the Domain Averaged Fermi Holes (DAFH) analysis as well as approaches based on the so-called generalized population analysis. In the latter approach the authors presented indices for electrons shared simultaneously in 3 or more atomic basins, which is very useful for formal 3-centre bonds but can be difficult to interpret with respect to partial multicentre bonding resulting from multiple contributions across a molecule. The source function ${ }^{[30]}$ has also been used to investigate the multicentre nature of various interactions at BCPs using QTAIM atomic basins. ${ }^{[31,32]}$

The DAFH approach presents a very generalized and powerful tool for investigating the delocalization over all molecular space of the electrons found within an atomic basin. Making 
use of some DAFH concepts, we recently developed a theoretical framework which, among other uses, can provide a real-space distribution of electrons delocalized between two atomic basins. ${ }^{[33]}$ In this work, we will introduce an extension of the Fragment, Atomic, Localized, Delocalized and Interatomic (FALDI) electron density decomposition scheme with which we can identify and quantify atom-pairs contributing significantly towards the formation of an AIL. This work does not aim to present another multicentre bond index. Rather, our approach allows for the investigation of the holistic, multicentre nature of the AIL itself by decomposing the total ED into contributions made by multiple atom-pairs. Finally, we note that our approach is fully applicable to any point $\mathbf{r}$ in molecular space; hence, it is also applicable to interacting regions where AILs are absent, as identified by the Non-Covalent Interaction (NCI) technique. ${ }^{[34]}$

\section{Computational details}

All structures were optimized in Gaussian 09, Rev. D., ${ }^{[35]}$ using B3LYP with Grimme's D3 empirical dispersion ${ }^{[36]}$ and the $6-311++\mathrm{G}(\mathrm{d}, \mathrm{p})$ basis set, in the gas phase. Coordinates of all optimized structures are given in PART 1 of the Supporting Information. QTAIM molecular graphs, as well as atomic overlap matrices, were calculated using AIMAll version 16.10.31. ${ }^{[37]}$ FALDI data were calculated using in-house software, and FALDI isosurfaces were visualized using VMD. ${ }^{[38]}$ We note that preliminary testing using multi-determinant wavefunctions within the Müller approximation ${ }^{[39]}$ give the same qualitative results. A 1electron implementation of FALDI for correlated wavefunctions should also be possible by following an approach reported by Cooper and Ponec. ${ }^{[40]}$ QTAIM and FALDI analyses of the two carbene complexes were done for structures in the singlet spin state only; the Fischer carbene complex is only stable in the singlet spin state while the Schrock carbene complex is approximately $10 \mathrm{kcal} \mathrm{mol}^{-1}$ less stable in the triplet spin state. Finally, our FALDI software is currently limited to smaller, Gaussian basis sets due to memory constraints. We intend to optimize it in the future to handle larger basis sets, e.g., as suggested by Bühl and Kabrede, ${ }^{[41]}$ in order to more accurately describe the transition metals, such as those used in this study.

\section{Theoretical background and development}

The recently introduced FALDI density decomposition scheme ${ }^{[33]}$ provides an exhaustive decomposition of the ED at any coordinate $\mathbf{r}$ in real-space into contributions from all of $M$ 
domains. These domains are usually chosen to be atomic basins as defined by QTAIM, ${ }^{[42]}$ yielding atomic contributions to the ED throughout an entire molecular system. While our previous work using FALDI $^{[33]}$ focused primarily on calculating and decomposing conformational deformation EDs, we focus here on the decomposition of the static $\mathrm{ED}$, i.e. the ED distribution of a single molecular state, regardless whether being at equilibrium or not.

\section{Domain Averaged Fermi Holes}

FALDI decomposes the total ED (tot-ED) at any point $\mathbf{r}$ into contributions from all atoms (domains),

$$
\rho(\mathbf{r})=\sum_{i}^{M} g_{i}(\mathbf{r})
$$

where $g_{i}(\mathbf{r})$ is the atomic ED (atom-ED) distribution associated with the $i$ th atomic basin. Calculation of $g_{i}(\mathbf{r})$ was originally developed by Ponec et al. and is a central concept from DAFH analysis. ${ }^{[43-46]} g_{i}(\mathbf{r})$ is defined by averaging the reference electron of the chargeweighted exchange-correlation (XC) electron hole over an atomic basin $\left(\Omega_{i}\right)$ :

$$
g_{i}\left(\mathbf{r}_{1}\right)=-\int_{i} \rho^{\text {Hole }}\left(\mathbf{r}_{1} ; \mathbf{r}_{2}\right) \rho\left(\mathbf{r}_{2}\right) d \mathbf{r}_{2}
$$

where $\rho^{\text {Hole }}\left(\mathbf{r}_{1} ; \mathbf{r}_{2}\right)=\rho\left(\mathbf{r}_{1}\right)-\rho^{\text {cond }}\left(\mathbf{r}_{1} ; \mathbf{r}_{2}\right)$ gives the reduced probability of ED at $\mathbf{r}_{1}$ as a result of an electron at $\mathbf{r}_{2}$, and $\rho^{\text {cond }}\left(\mathbf{r}_{1} ; \mathbf{r}_{2}\right)=\frac{2 \rho_{2}\left(\mathbf{r}_{1}, \mathbf{r}_{2}\right)}{\rho\left(\mathbf{r}_{2}\right)}$ gives the conditional probability of finding an electron at $\mathbf{r}_{1}$ given an electron at $\mathbf{r}_{2}$. The $\mathrm{XC}$ hole provides the origin of the excluded electron at $\mathbf{r}_{1}$, and therefore can be used as a pseudo-dynamic distribution of an electron within volume element $d \mathbf{r}_{2}$. Charge-weighting the XC hole by $\rho\left(\mathbf{r}_{2}\right)$ (as in Eq. 2) then gives the molecular-wide distribution of all electrons found on average in $d \mathbf{r}_{2}$.

An atom-ED distribution provides the distribution throughout all molecular space of the electrons that are found, on average, within a particular atomic basin. Integrating $g_{i}(\mathbf{r})$ over all molecular space yields the QTAIM-defined atomic population, $N\left(\Omega_{i}\right)$, and is equivalent to $N\left(\Omega_{i}\right)$ obtained by integrating the tot-ED over an atomic basin, $\Omega_{i}$. The atom-ED distributions and the QTAIM atomic basins therefore describe the same electrons, but where QTAIM produces non-overlapping, $1^{\text {st }}$-order atomic domains, atom-ED distributions produce fuzzy, pseudo- $2^{\text {nd }}$-order fields due to the inclusion of XC effects. In order to avoid the expensive integration of the pair density over multiple atomic basins, $g_{i}(\mathbf{r})$ is usually calculated through elements of atomic overlap matrices (AOM): 


$$
g_{i}\left(\mathbf{r}_{1}\right)=2 \sum_{\lambda \sigma}^{N} \chi_{\lambda}\left(\mathbf{r}_{1}\right) \chi_{\sigma}\left(\mathbf{r}_{1}\right) S_{\sigma \lambda}^{i}
$$

where

$$
S_{\sigma \lambda}^{i}=\left\langle\chi_{\sigma} \mid \chi_{\lambda}\right\rangle_{i}=\int_{i} \chi_{\sigma}\left(\mathbf{r}_{1}\right) \chi_{\lambda}\left(\mathbf{r}_{1}\right) d \mathbf{r}_{1}
$$

$S_{\sigma \lambda}^{i}$ is an element of the AOM associated with a specific domain $\Omega_{i}$, and $\chi(\mathbf{r})$ are natural molecular orbitals (which reduce to canonical molecular orbitals in single-determinant wavefunctions).

The procedure of a full DAFH analysis includes the diagonalization and subsequent isopycnic transformation of the AOMs in order to produce domain-localized natural orbitals associated with each DAFH function. ${ }^{[45]}$ However, our approach uses a different decomposition of each DAFH function, related to QTAIM atomic populations, as discussed next.

\section{The Fragment, Atom, Localized, Delocalized and Interaction Density Decomposition}

FALDI decomposes each atom-ED into two components, i.e. the density that is localized to a specific atomic basin (loc-ED, $\left.\mathcal{L}_{i}(\mathbf{r})\right)$ and the density that is delocalized by two atomic basins (deloc-ED, $\left.\mathcal{D}_{i, j}(\mathbf{r})\right)$, as shown in Eq. 5:

$$
g_{i}(\mathbf{r})=\mathcal{L}_{i}(\mathbf{r})+\sum_{j \neq i}^{M} \frac{1}{2} \mathcal{D}_{i, j}(\mathbf{r})
$$

FALDI-generated loc-ED distributions and atom-pair deloc-ED distributions are real-space, i.e. 3D distributions of the QTAIM-defined electron localization index (LI, $\lambda\left(\Omega_{i}\right)$ ) and electron delocalization index (DI, $\delta\left(\Omega_{i}, \Omega_{j}\right)$ ), respectively. loc-ED and deloc-ED distributions are calculated similarly to atom-ED distributions:

$$
\begin{gathered}
\mathcal{L}_{i}(\mathbf{r})=2 \sum_{\lambda \sigma}^{N} \chi_{\lambda}(\mathbf{r}) \chi_{\sigma}(\mathbf{r})\left(\mathbf{S}^{i} \mathbf{S}^{i}\right)_{\sigma \lambda} \\
\mathcal{D}_{i, j}(\mathbf{r})=2 \sum_{\lambda \sigma}^{N} \chi_{\lambda}(\mathbf{r}) \chi_{\sigma}(\mathbf{r})\left(\mathbf{S}^{i} \mathbf{S}^{j}\right)_{\sigma \lambda}
\end{gathered}
$$

where $\left(\mathbf{S}^{i} \mathbf{S}^{i}\right)_{\sigma \lambda}$ and $\left(\mathbf{S}^{i} \mathbf{S}^{j}\right)_{\sigma \lambda}$ are elements of the matrix products of the AOMs associated with domain $\Omega_{i}$ with itself and domain $\Omega_{i}$ with $\Omega_{j}$, respectively. Note that $\operatorname{LI}\left(\Omega_{i}\right)=\operatorname{tr}\left(\mathbf{S}^{i} \mathbf{S}^{i}\right)$, and $0.5 \mathrm{DI}\left(\Omega_{i}, \Omega_{j}\right)=\operatorname{tr}\left(\mathbf{S}^{i} \mathbf{S}^{j}\right)$. Integration of a loc-ED or deloc-ED distribution over all space gives the corresponding QTAIM-defined LI or DI term, respectively. Historically, the 
$\operatorname{LI}\left(\Omega_{i}\right)$ and $\operatorname{DI}\left(\Omega_{i, j}\right)$ terms have been interpreted as the number of electrons that are localized to the $i$ th basin and the number of electrons that are delocalized between the $i$ th and $j$ th basins, respectively. ${ }^{[47-49]}$ This interpretation implies that $\operatorname{LI}\left(\Omega_{i}\right)$ is a measure of the number of electrons found exclusively in $\Omega_{i}$. However, due to averaging involved during the calculation of LI by QTAIM, such an interpretation is not correct, which we will elaborate on thoroughly in near future. Due to this general interpretation with regards to LI, which we do not agree with, we focus here on exploring multicentre character of AILs from only the deloc-ED distributions.

Deloc-ED distributions $\left(\mathcal{D}_{i, j}(\mathbf{r})\right)$ can be interpreted as the probability of finding an electron at any coordinate $\mathbf{r}$ which is simultaneously correlated with two different atomic basins. 3D isosurfaces of $\mathcal{D}_{i, j}(\mathbf{r})$ that are calculated from FALDI analyses therefore illustrate the molecular-wide distribution of electrons which can statistically be found in both the $i$ th and $j$ th atomic basins. These deloc-ED distributions are directly related to the magnitude of the interatomic XC energy, with increasing interatomic delocalization resulting in more stabilizing XC effects. DIs and deloc-ED distributions can be used as a measure of the covalency of an interaction, and DI is often used as an indication of the bond order between two atoms connected by an AIL. ${ }^{[47-49]}$ DI is usually calculated by considering the mutual overlap of MOs across two atomic basins, and is therefore a very good link between MOtheory and Quantum Chemical Topology (QCT). ${ }^{[49]}$ The full sets of LIs and DIs in a molecule have been combined as localization-delocalization matrices (LDMs) by Matta et al. ${ }^{[50]}$ This approach has been used to understand the complex network of localized and delocalized contributions to atomic populations. ${ }^{[51,52]}$ While an LDM provides a very valuable condensed overview of ED localization and delocalization at an atomic level, it does not provide valuable chemical information inherent in the real-space distributions of correlated ED. To our knowledge, FALDI is the first technique able to calculate the distribution of DI in realspace and we utilise this feature in this study to show that the manner in which electrons are delocalized is equally as important, and more informative than just the amount of delocalized electrons.

There is, however, a fundamental difference between the DI term and deloc-ED distribution. DI is always an integrated, positive value. On the other hand, deloc-ED distributions can be either a positive or negative values at a specific point $\mathbf{r}$. Regions where $\mathcal{D}_{i, j}(\mathbf{r})>0$ represent a constructive interference of MOs simultaneously overlapping with two atomic basins $\Omega_{i}$ and $\Omega_{j}$ at $\mathbf{r}$, indicate regions in $3 \mathrm{D}$ space where electrons are positively 
correlated between both basins. Such a constructive overlap results in an increase of the totED. Regions where $\mathcal{D}_{i, j}(\mathbf{r})<0$, on the other hand, are due to deconstructive interference at $\mathbf{r}$ of MOs overlapping with two atomic basins, indicate the regions in space where electrons are negatively correlated between the basins, thereby reducing the tot-ED.

\section{Partial second derivatives of deloc-ED distributions}

Our FALDI decomposition produces a large set of deloc-ED distributions. Each deloc-ED is a component of the tot-ED (hence, a contribution made by a specific atom-pair). However, only some components of the tot-ED will contribute to the formation of an AIL. This brings us to the primary objective of this work: isolating the components of the tot-ED that contribute to the formation of a specific AIL, as well as quantifying and visualizing their contributions. Each point of an AIL has the specific property that ED is concentrated perpendicular to the AIL (as measured by the second eigenvalue of the Hessian matrix, $\lambda_{2}$ ). Therefore, the second derivative (and more specifically, $\lambda_{2}$ ) of any deloc-ED distribution can be determined at any given point $\mathbf{r}$. In doing so, one can precisely determine whether a particular contribution made by a specific atom-pair is concentrating (negative partial $2^{\text {nd }}$ derivative) or depleting (positive partial $2^{\text {nd }}$ derivative) the tot-ED. Deloc-ED distributions that are concentrating the tot-ED therefore facilitate the formation of an AIL, whereas delocED distributions that are depleting the tot-ED hinder the formation of an AIL. One must also realize that a specific component of deloc-ED, e.g. $\mathcal{D}_{i, j}(\mathbf{r})$, might be concentrating in the interatomic region of $\Omega_{i}$ and $\Omega_{j}$ but depleting in another, e.g. in the interatomic region of $\Omega_{i}$ and $\Omega_{k}$. The FALDI-based decomposition of the total density distribution of a molecular state implemented in this work is shown in Scheme 1.

In the orthodox MO theory, each orbital can be classified in terms of either a bonding, nonbonding or antibonding contribution with respect to a particular chemical interaction; the effects of (de)constructive interference on both the binding energies and electron density concentration have been well documented and can be found in any textbook on electronic structure. Similarly, the sign of the partial second derivative of a deloc-ED distribution is a result of constructive or deconstructive interference of MOs overlapping simultaneously across two atomic basins. Therefore, in analogy to MO bonding theory, we label each atompair's deloc-ED distribution accordingly, i.e. an atom-pair contribution to the total deloc-ED as either bonding, nonbonding or antibonding based on whether it concentrates (bonding), 
depletes (nonbonding) or reduces (antibonding) ED in a specific region of space, as illustrated in Scheme 2.

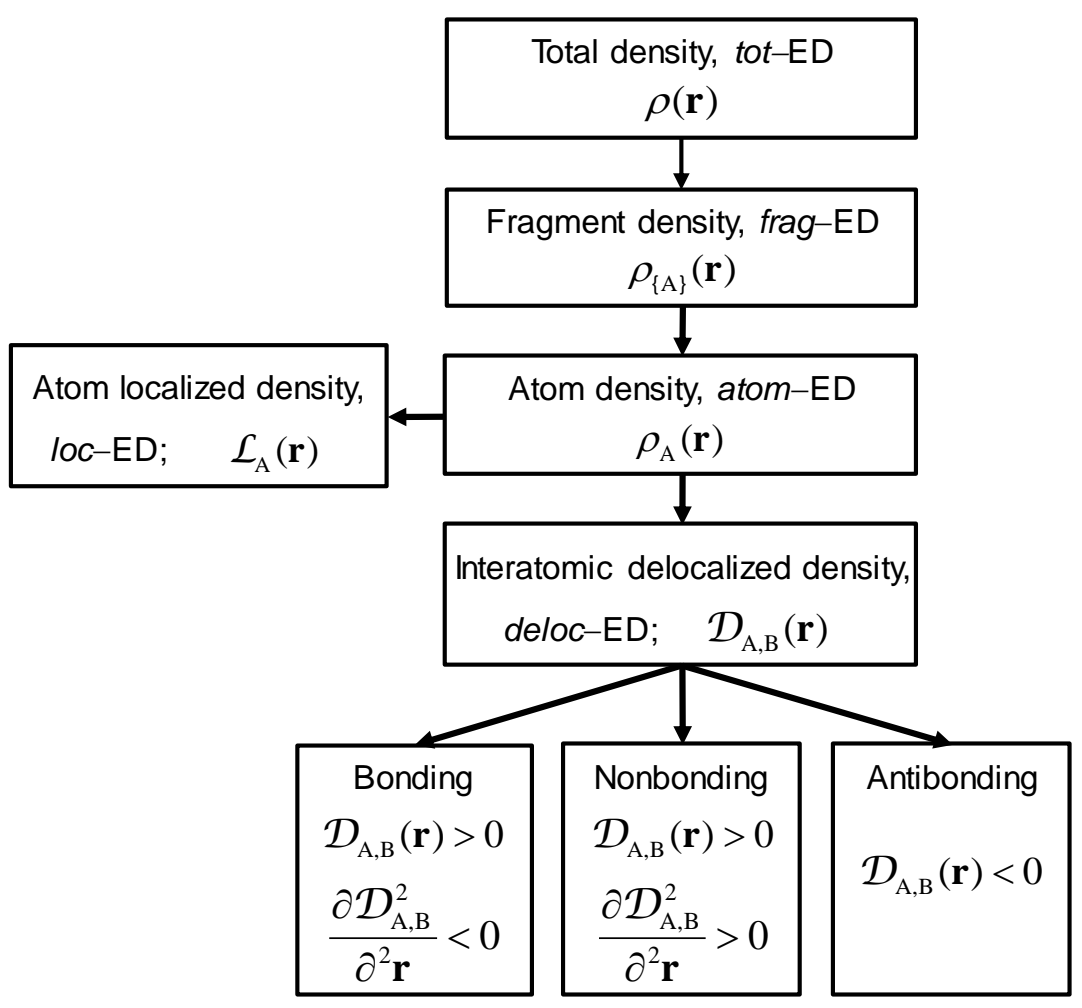

Scheme 1. FALDI-based decomposition of the total static ED (i.e., the ED distribution of a single molecular state) implemented in this work.
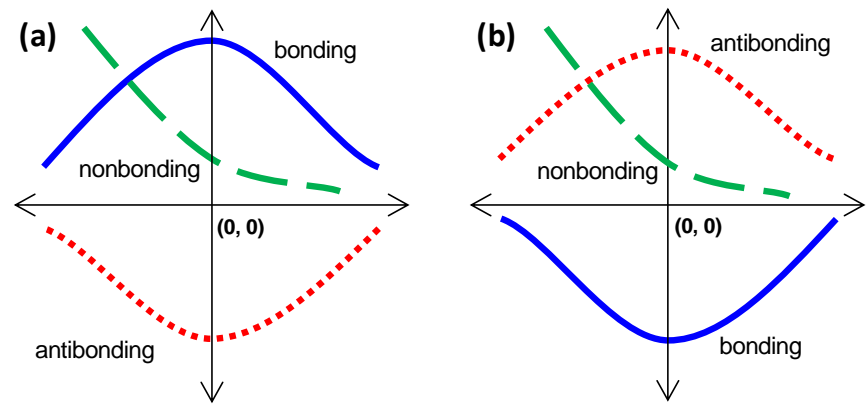

Scheme 2 (a) Examples of the cross-sections illustrating trends of bonding, nonbonding or antibonding ED distributions (computed for a selected atom-pair) as well as (b) their $2^{\text {nd }}$ partial derivatives.

The above classification of each deloc-ED contribution to the tot-ED allows for the identification of atom-pairs and quantification of their contributions in the formation of a particular AIL (bonding deloc-ED), as well as of the atom-pairs that hinder formation of the AIL (nonbonding and antibonding contributions to the total deloc-ED). The presence of multiple contributions towards bond formation also allows us to extend the connectivity 
allowed by an AIL to more than just two atoms, a conceptual development which can have wide-ranging implications. Specifically, in our approach each AIL can be interpreted as the net effect of a number of bonding and competing (non- and antibonding) contributions. Hence, the presence of an AIL must imply that bonding deloc-ED distributions dominate.

We note that Gatti and Baders, source function $(\mathrm{SF})^{[30]}$ also provides a full decomposition of $\rho(\mathrm{r})$ at any given $\mathbf{r}$ into contributions from all atomic basins, thereby satisfying Eq. 1. The SF has been used to interpret various chemical phenomena, ${ }^{[31,32]}$ including multicentre contributions at bond-critical points, ${ }^{[31]}$ from a QTAIM-based perspective in real-space. The second derivatives of the various atomic SFs in a molecule can therefore be calculated and classified according to how each atom facilitates or hinders the formation of an AIL that, in some respect, is similar to the approach discussed above. The SF, which is a Green's function based on the Laplacian of the total ED, has the advantage that it is quite quick to calculate, also from experimental densities. One must stress, however, that in terms of atom-pair contributions and explicit exchange-correlation effects it provides less information than the FALDI approach. Clearly, it would be of great interest and importance to explore advantages (limitations) of to both, SF- and FALDI-based, approaches and we plan to embark on such studies in near future.

\section{Results and discussion}

We aim to determine the multicentre nature of the AILs in five 'classical' bonding interactions from a delocalised ED perspective. Moreover, we hope to provide convincing evidence that (i) observed BCPs from tot-ED topological analyses, as obtained from QTAIM, should not be simply viewed or interpreted as an indication of a favourable bonding interaction between two atoms alone and (ii) the emergence of a $\mathrm{BCP}$, and therefore the nature of an interaction (between the atoms linked by the associated AIL) depends on contributing (bonding) deloc-ED coming from other/distant atoms in a molecular system.

The first structure that we selected to investigate is the lowest energy conformer of $\beta$ alanine (1 in Figure 1) as it presents a very good example of a multicentre, intramolecular bonding interaction. QTAIM calculations of $\mathbf{1}$ show that an AIL is present between hydrogen atom $\mathrm{H} 6(\mathrm{H})$ and nitrogen atom $\mathrm{N} 11(\mathrm{Y})$ and thus $\mathrm{BCP}(\mathrm{H} 6, \mathrm{~N} 11)$. This molecular graph alone does not provide any information regarding the multicentre bonding nature of this interaction. Moreover, it does not provide evidence to suggest how other atoms, such as O5 (X), C8 (Z) or $\mathrm{C} 1(\mathrm{~W})$, influence (i.e. by either facilitating or hindering) the emergence of AIL(H6,N11). 
In order to compare with $\mathbf{1}$, we decided to investigate the interactions of two systems that, relative to each other, should display quite opposing degrees of multicentre bonding nature. The interaction between a boron (B1) and hydrogen (H7) atom in diborane, 2 in Figure 1, is well-known as consisting of a large degree of multicentre character. ${ }^{[2-6]}$ On the other hand, the interaction between carbon atoms $\mathrm{C} 9$ and $\mathrm{C} 12$ in $n$-butane, 3 in Figure 1, which is
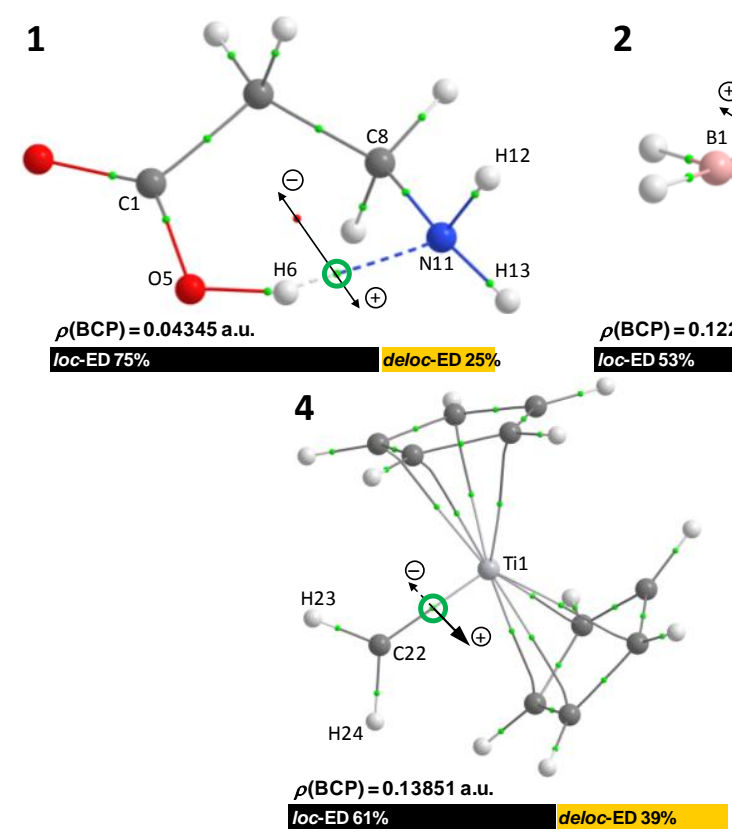
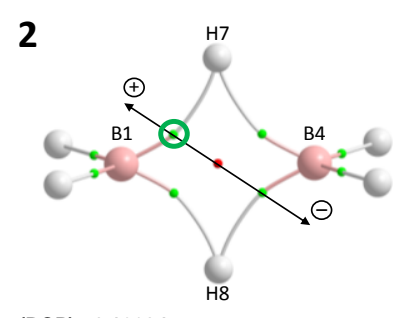
deloc-ED $47 \%$ 5

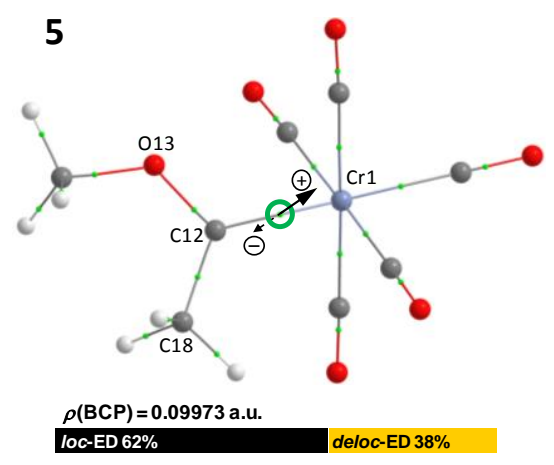

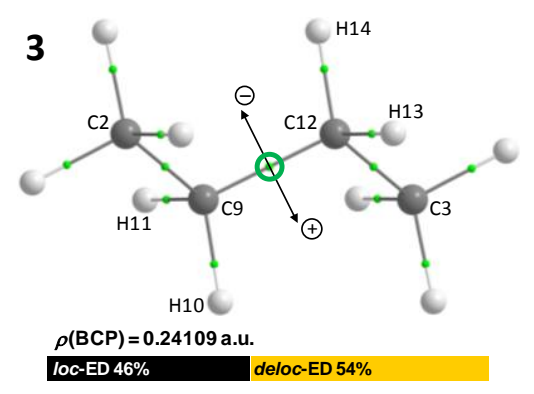

Figure 1 Molecular graphs of $\beta$-alanine (1), diborane (2), 'linear' $n$-butane (3), Schrock, $\left[(\mathrm{Cp})_{2} \mathrm{Ti}=\mathrm{CH}_{2}\right]$ (4) and Fischer, $\left[(\mathrm{CO})_{5} \mathrm{Cr}=\mathrm{C}\left(\mathrm{OCH}_{3}\right) \mathrm{CH}_{3}\right]$ (5) carbene complexes, including the vectors along which $\mathrm{ED}$ decomposition analyses are done. Encircled (green) are the BCPs at which the multicentre bonding nature of an interaction is explored. Tot-ED $(\rho)$ and percentage of loc-ED and deloc-ED at these BCPs is given directly below the relevant molecular graphs. All $(+3,+1)$ and $(+3,+3)$ critical points in $\mathbf{4}$ have been omitted for clarity.

representative of a classic single covalent $\mathrm{C}-\mathrm{C}$ bond, provides an example of a bicentric interaction. We therefore expect that our ED decomposition scheme should be able to clearly distinguish between the multicentre natures of these two interactions, as well as agree qualitatively with that reported in the literature.

Lastly, we decided to compare the nature of two different metal-carbon (M-C) carbene bonding interactions, i.e. one of a typical Schrock carbene complex, $\left[(\mathrm{Cp})_{2} \mathrm{Ti}=\mathrm{CH}_{2}\right]$, and one of a typical Fischer carbene complex, $\left[(\mathrm{CO})_{5} \mathrm{Cr}=\mathrm{C}\left(\mathrm{OCH}_{3}\right) \mathrm{CH}_{3}\right]$. The molecular graphs of the two complexes are shown in Figure 1 (4 and 5, respectively). In the case of 5, oxygen atom $\mathrm{O} 13$ is expected to yield an additional degree of deloc-ED of a bonding nature to the carbene bond, since such heteroatoms are known to facilitate $\pi$-backbonding. ${ }^{[21]}$ As a consequence, this $\mathrm{M}-\mathrm{C}$ carbene bonding interaction is expected to have more multicentre bonding character 
as compared to its counterpart in $\mathbf{4}$ (no heteroatom), even though the magnitude of the tot-ED at $\mathrm{BCP}(\mathrm{Cr} 1, \mathrm{C} 12)$ in 5 ( $\rho=0.09973$ a.u. $)$ is considerably less than at $\mathrm{BCP}(\mathrm{Ti1}, \mathrm{C} 22)$ in 4 ( $\rho=$ 0.13851 a.u.). In the following sections we discuss results obtained from the ED decomposition along the indicated vectors whereas quantitative analyses (decomposition of either total or delocalized EDs) are reported at BCPs, as indicated in Figure 1.

\subsection{Intramolecular $\mathrm{H}$-bonding interaction in $\beta$-alanine}

Fig. 2(a) shows the FALDI-based decomposition of the tot-ED (silver) into ED contributions that are localized (black) and delocalized (gold). We note that both trends have a local maximum in the vicinity of $\mathrm{BCP}(\mathrm{H} 6, \mathrm{~N} 11)$ and a local minimum close to the RCP (small red sphere, 1 in Figure 1). The decomposition of the total deloc-ED, Figure 2(b), yields the sum totals of all contributions that are of a bonding (blue line), nonbonding (green line) and antibonding (red line) nature with respect to $\mathrm{BCP}(\mathrm{H} 6, \mathrm{~N} 11)$.

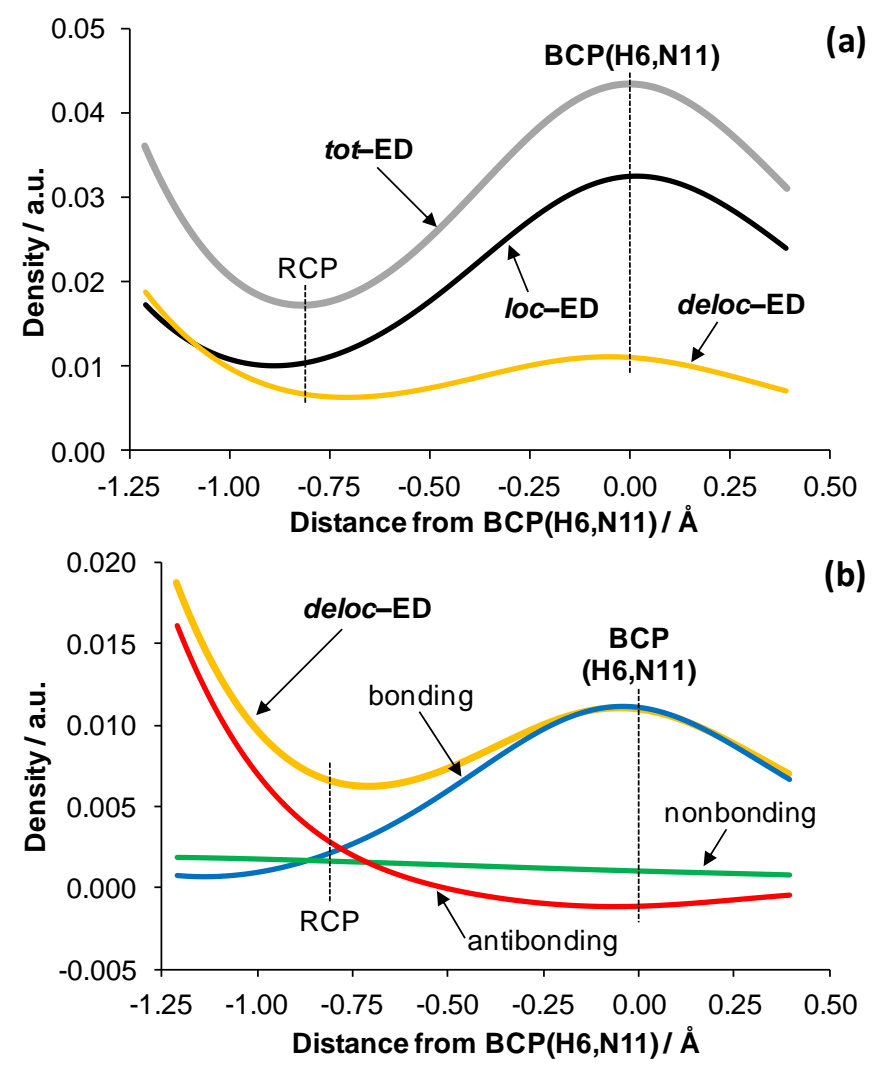

Figure 2. (a) FALDI-based decomposition of the tot-ED (silver) into loc- (black) and deloc-ED (gold) in $\beta$-alanine along the vector indicated in Figure 1. (b) Subsequent FALDI-based decomposition of the deloc-ED into constructive (blue, bonding nature), nonconstructive (green, nonbonding nature) and deconstructive (red, antibonding nature) electron correlation with respect to the $\mathrm{BCP}(\mathrm{H} 6, \mathrm{~N} 11)$. 
Fig. 2 (b) clearly illustrates that the total deloc-ED (0.01102 a.u.) consists of a significant quantity of ED of a bonding nature (0.01107 a.u.) and it is the only one, among the three types of contributions that directly contributes towards the emergence of $\mathrm{BCP}(\mathrm{H} 6, \mathrm{~N} 11)$. Nonbonding and antibonding deloc-ED components are approximately equal in magnitude but of a different sign, at 0.00104 and -0.00109 a.u., respectively.

Atom-pair O5,N11, i.e. atoms $\mathrm{X}$ and $\mathrm{Y}$, is mainly responsible for the overall deconstructive electron correlation at $\mathrm{BCP}(\mathrm{H} 6, \mathrm{~N} 11)$. It accounts for -0.00079 a.u. (72\%) of the total antibonding deloc-ED, where it is also a local minimum along the analysed vector. Of all five the structures studied in this work, $\beta$-alanine is the only one to show a significant quantity of deloc-ED of an antibonding nature at BCP of interest (see PART 2 of the Supporting Information for deloc-ED decomposition graphs pertaining to the other four structures).

Figure 3 illustrates the 3D-isosurfaces of the major (either summed or individual) contributions to the total deloc-ED that are of a bonding, nonbonding or antibonding nature with respect to $\mathrm{BCP}(\mathrm{H} 6, \mathrm{~N} 11)$. These three real-space $\mathrm{ED}$ distributions clearly illustrate how a particular atom-pair in the molecule either (i) concentrates, Figure 3(a), (ii) depletes, Figure 3(b), (but concentrates ED elsewhere in the molecule), or (iii) specifically reduces, Figure $3(\mathbf{c}), \mathrm{ED}$ in the vicinity of $\mathrm{BCP}(\mathrm{H} 6, \mathrm{~N} 11)$.

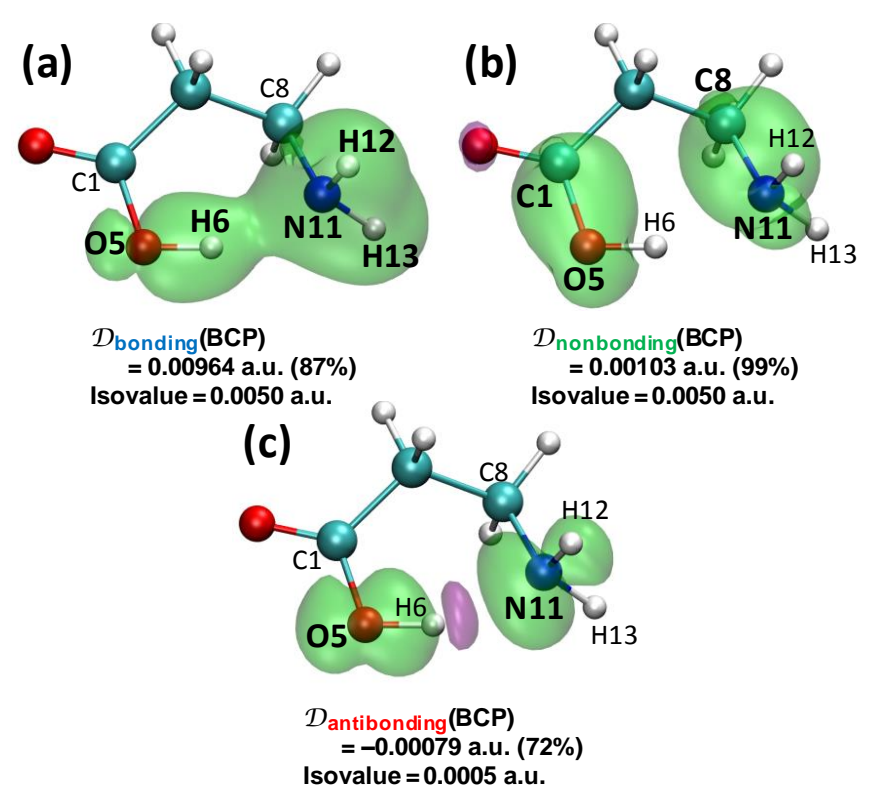

Figure 3. Deloc-ED 3D-isosurfaces of the major constructive (a, $87 \%$ of bonding ED, comprising $(\mathrm{O} 5, \mathrm{H} 6)+(\mathrm{H} 6, \mathrm{~N} 11)+(\mathrm{N} 11-\{\mathrm{H} 12, \mathrm{H} 13\}))$, nonconstructive $(\mathbf{b}, 99 \%$ of nonbonding ED, comprising $(\mathrm{C} 1, \mathrm{O} 5)+(\mathrm{C} 8, \mathrm{~N} 11))$ and deconstructive $(\mathbf{c}, 72 \%$ of antibonding $\mathrm{ED}$, comprising $(\mathrm{O} 5, \mathrm{~N} 11))$ electron correlation contributing factors with respect to $\mathrm{BCP}(\mathrm{H} 6, \mathrm{~N} 11)$ in $\beta$-alanine. Colour coding: green $=$ positive, purple $=$ negative . 
Figure 3(a) reveals how the atom-pairs' contributions collectively form a channel of constructive electron correlation between the nuclei involved in the intramolecular interaction, thereby naturally facilitating the emergence of $\mathrm{BCP}(\mathrm{H} 6, \mathrm{~N} 11)$. Deloc-ED of a nonbonding or antibonding nature in this interatomic region, Figure 3 (b and c, respectively), both naturally hinder the emergence of this BCP and can therefore be classified as 'competing secondary interactions' according to the nomenclature of Tognetti et al. ${ }^{[53,54]}$ Of the atom-pairs that deplete deloc-ED in the vicinity of $\mathrm{BCP}(\mathrm{H} 6, \mathrm{~N} 11)$, Figure 3(b), C1, O5 and C8,N11 are the largest in magnitude; their constructive electron correlation is mainly distributed all along their own covalent bonds, i.e. $\mathrm{C} 1-\mathrm{O} 5$ and $\mathrm{C} 8-\mathrm{N} 11$, respectively.

Since our study focuses on the multicentre nature of a particular AIL, we further decompose the total deloc-ED of a bonding nature (blue line in Figure 2(b)). In doing so, we identify and quantify the major contributing atom-pairs that concentrate ED at BCP(H6,N11).

From Figure 4(a) we note that atom-pair H6,N11 (atoms H and Y), i.e. the two atoms linked by the AIL (which we will henceforth refer to as the primary interaction), does not make the largest contribution to the deloc-ED of a bonding nature.

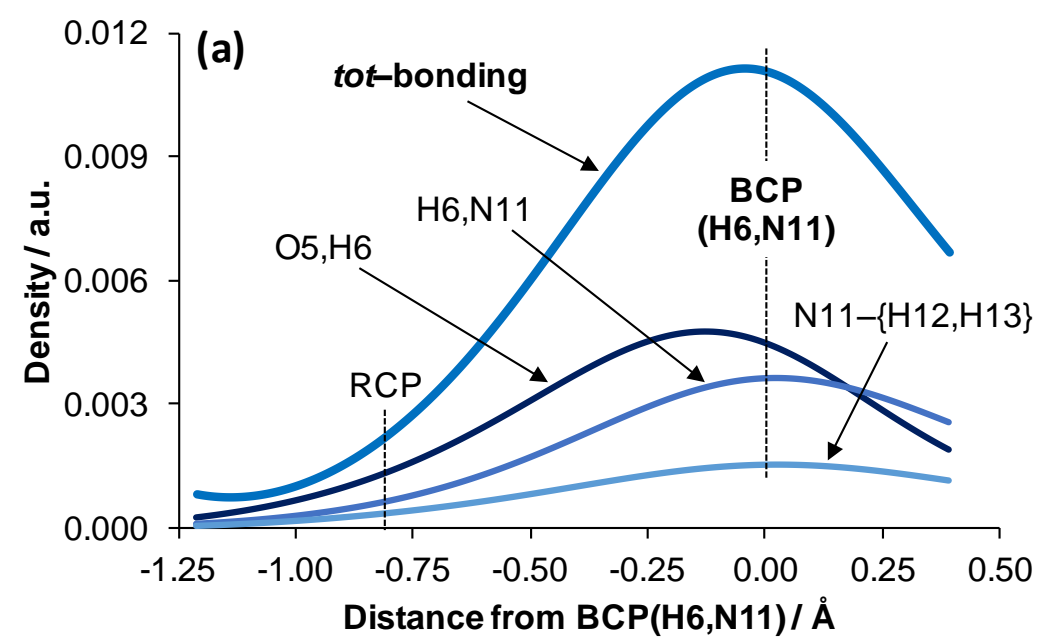

(b)

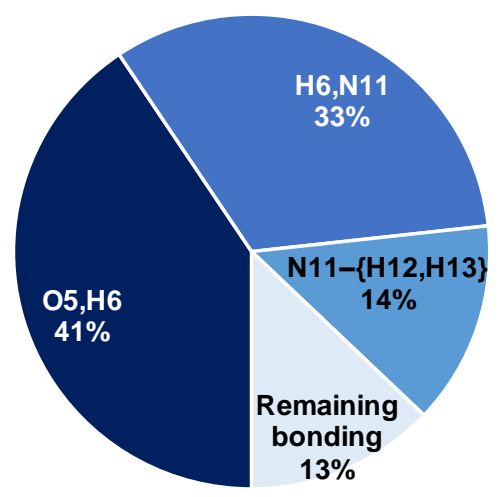

Figure 4. (a) FALDI-based decomposition, along the indicated vector for $\mathbf{1}$ in Figure 1, of the constructive electron correlation (blue line in Figure 2(b), bonding nature) with respect to the $\mathrm{BCP}(\mathrm{H} 6, \mathrm{~N} 11)$ in $\beta$-alanine, into the major contributing atom-pairs. (b) Pie-chart summarising percentage-wise the major contributing atom-pairs at the $\mathrm{BCP}(\mathrm{H} 6, \mathrm{~N} 11)$.

Only one-third (see the pie chart in Figure 4(b)) of the total constructive deloc-ED at $\mathrm{BCP}(\mathrm{H} 6, \mathrm{~N} 11)$ is made by the primary interaction. From the FALDI-based decomposition

¥ In a simulated aqueous phase (using implicit solvent model PCM) the intramolecular $\mathrm{H}$-bonding distance ( $\mathrm{H} 6 \cdots \mathrm{N} 11)$ is much shorter and the largest contribution to the deloc-ED of a bonding nature is from the atompair corresponding to the primary interaction, i.e. H6,N11. The same phenomena has been observed using the $S F .{ }^{[32]}$ 
data, the major contribution to the deloc-ED of a bonding nature is in fact from atom-pair O5,H6 (atoms $\mathrm{X}$ and $\mathrm{H}$ ), which accounts for $41 \%$ of the total. The third largest contribution comes from N11,H12 and N11,H13 atom-pairs. Each one contributes $7 \%$ to the total and thus a collective sum of $14 \%$ is yielded by the N11- $\mathrm{H} 12, \mathrm{H} 13\}$ 'group'. All other atom-pairs made rather smallcontributions - we therefore summed their individual values together to get the 'Remaining bonding' percentage, which is $13 \%$ of the total, Figure $4(\mathbf{b})$. The FALDIbased decomposition of the deloc-ED at $\mathrm{BCP}(\mathrm{H} 6, \mathrm{~N} 11)$ has revealed quantitatively (percentage-wise) the multicentre bonding nature of the intramolecular H-bonding interaction in $\beta$-alanine. Furthermore, we note that atom-pair combinations involving the three atoms $\mathrm{O5}$ (X), H6 (H) and N11 (Y) account for the vast majority of deloc-ED of a bonding nature. This is an important result, not only in terms of quantifying the multicentre character (in this context, we can now approximate this interaction as three-centre), but also in that the result is in full agreement with IUPAC's basic recommendation ${ }^{[17,18]}$ of depicting an H-bond to comprise a series of at least three chemically-bonded atoms, namely atoms $\mathrm{X}-\mathrm{H} \cdots \mathrm{Y}$.

Fig. 5 shows the 3D-isosurfaces of the three major bonding contributions (see pie chart in Figure 4(b)) to the total deloc-ED; it is clear that all of them concentrate ED in the vicinity of $\mathrm{BCP}(\mathrm{H} 6, \mathrm{~N} 11)$.
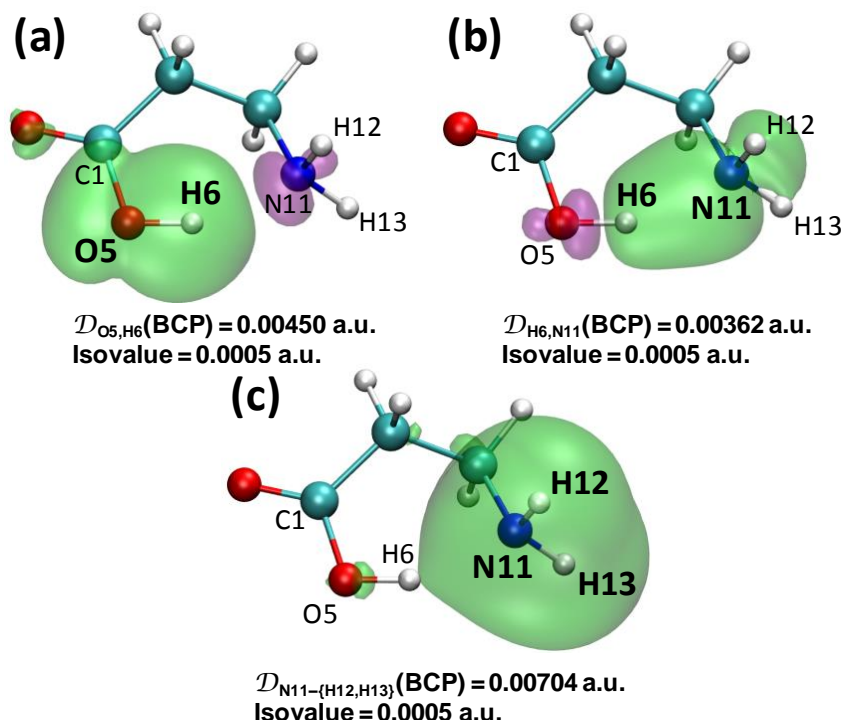

Figure 5. Deloc-ED 3D-isosurfaces of the major constructive electron correlation contributing atompairs with respect to the $\mathrm{BCP}(\mathrm{H} 6, \mathrm{~N} 11)$ in $\beta$-alanine: (a) $\mathcal{D}_{\mathrm{O} 5, \mathrm{H} 6}(41 \%)$, (b) $\mathcal{D}_{\mathrm{H} 6, \mathrm{~N} 11}(33 \%)$ and (c) $\mathcal{D}_{\mathrm{N} 11-\{\mathrm{H} 12, \mathrm{H} 13\}}(14 \%)$. Colour coding: green $=$ positive, purple $=$ negative .

The primary interaction, Figure 5(b), forms a very clear channel of constructive electron correlation all along AIL(H6,N11). This particular isosurface may be related to the so-called 'privileged exchange-channel' as per thinking by Pendás et al. ${ }^{[5]}$ However, the maximum 
value for this atom-pair does not occur at the same coordinate as the BCP along the analyzed vector, Figure 4(a), indicating that the overall or resultant 'privileged exchange-channel' (i.e. the AIL) is the sum of all constructive electron correlation contributions and hence does not coincide with a simple two-atom interpretation of a BCP. Counter intuitively, the electrons shared by $\mathrm{O} 5$ and H6 (Figure 5(a)), as well as the electrons shared by N11 and its bonded hydrogens (Fig. 5 (c)), extend into the H6,N11 interatomic region along the AIL. These three FALDI-based isosurfaces, when considered individually and then as a collective group (as shown in Figure 3(a)), provide a qualitative understanding of the multicentre nature of the AIL between atoms $\mathrm{H} 6$ and N11 in $\beta$-alanine.

\subsection{Multicentre bonding nature of a boron-hydrogen interaction in diborane}

Figures 6(a and b) show results obtained for the constructive deloc-ED distributions in diborane with respect to $\mathrm{BCP}(\mathrm{B} 1, \mathrm{H} 7)$. Additional FALDI decompositions are included in PART 2 of the Supporting Information. The largest contribution to the bonding deloc-ED comes from the atom-pair linked by the AIL, B1 and H7 (the primary interaction), and accounts for one-third (33\%) of the total. Interestingly, the ED that is delocalized between the other boron atom with the same hydrogen, i.e. atom-pair B4, $\mathrm{H} 7$, contributes approximately the same amount $(31 \%)$ of bonding deloc-ED. These two B,H atom-pairs therefore contribute almost equally to $\mathrm{BCP}(\mathrm{B} 1, \mathrm{H} 7)$ and (due to symmetry) $\mathrm{BCP}(\mathrm{B} 4, \mathrm{H} 7)$.

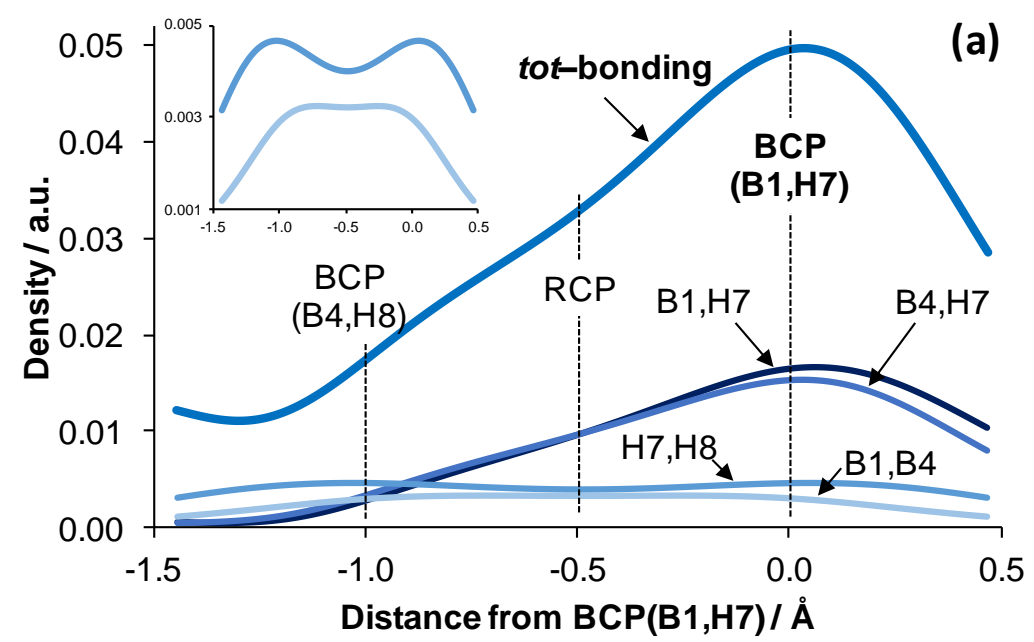

(b)

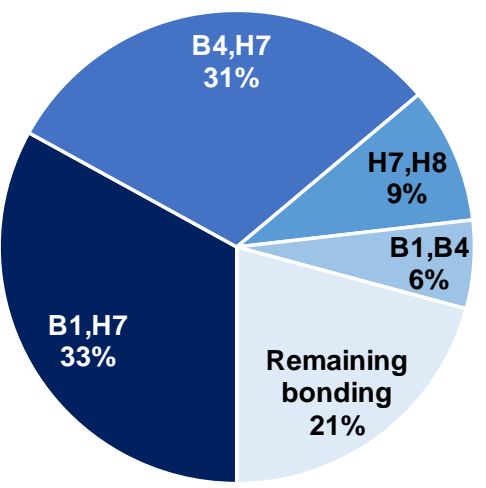

Figure 6. (a) FALDI-based decomposition of the constructive electron correlation (i.e. deloc-ED of a bonding nature) with respect to $\mathrm{BCP}(\mathrm{B} 1, \mathrm{H} 7)$ in diborane, along the indicated vector for $\mathbf{2}$ in Figure 1, into the major atom-pair contributions. Inset: magnified trends for atom-pairs $\mathrm{H} 7, \mathrm{H} 8$ and B1,B4. (b) Pie-chart summarizing percentage-wise the major contributing atom-pairs at the $\mathrm{BCP}(\mathrm{B} 1, \mathrm{H} 7)$. 
Not only is this result an indication of a 3-centre B-H-B interaction but it is also to a large extent consistent with the picture of $3 \mathrm{c} 2 \overline{\mathrm{e}}$ bonding. ${ }^{[6]}$ The third and fourth largest contributions are from atom-pairs that are not directly linked to each other with an AIL, namely $\mathrm{H} 7, \mathrm{H} 8(9 \%)$ and B1,B4 (6\%). These two atom-pairs, together with the remainder of the constructive deloc-ED, collectively account for $\sim 36 \%$ of the total amount at $\mathrm{BCP}(\mathrm{B} 1, \mathrm{H} 7)$. This is quite a substantial figure, indicating that the B1-H7 bonding interaction displays considerably more multicentre character than what is suggested by the rather oversimplified $3 \mathrm{c} 2 \overline{\mathrm{e}}$ model. This finding supports earlier results by Ponec and Uhlik ${ }^{[56]}$ who suggested that delocalized 3-centre interactions require significant (non-vanishing) 2-centre delocalization between all atom-pairs involved. Due to the symmetry $\left(D_{2 h}\right)$ of this diborane structure, the same observations apply to the remaining three B-H interactions involving $\mathrm{H} 7$ and $\mathrm{H} 8$.

Figure 7 illustrates the 3D-isosurfaces computed for four atom-pairs that made major contributions of bonding nature to the total deloc-ED at $\mathrm{BCP}(\mathrm{B} 1, \mathrm{H} 7)$. The deloc-ED distributions for atom-pairs B1,H7 and B4,H7 are remarkably similar, Figure 7 (a and b). Both distributions extend fully in-between the atomic basins of B1, H7 and B4, clearly indicating the tricentric manner in which electrons are shared across these centres. Note that Ponec et al. ${ }^{[27-29]}$ have previously shown (within the framework of DAFH) that a boron atom's ED distribution takes place across the $\mathrm{B} \cdots \mathrm{H} \cdots \mathrm{B}$ region. However, FALDI clearly shows the three-centre nature of each $\mathrm{B}-\mathrm{H}$ delocalized channel.

The ED that is delocalized between atom-pair H7,H8 occurs largely through the associated $\mathrm{B}, \mathrm{H}$ BCPs (explaining why this atom-pair contributes to deloc-ED concentration at all four $\mathrm{BCPs}$ related to $\mathrm{B} 1, \mathrm{~B} 4, \mathrm{H} 7$ and $\mathrm{H} 8$ ), as well as through a direct channel between their own nuclei, Figure 7(c). Finally, the manner in which electrons are shared between atoms B1 and B4 occurs primarily through the channel between atoms $\mathrm{H} 7$ and H8, Figure 7(d). This is quite an unexpected finding, revealing that boron atoms share ED 'through bond' (i.e. by means of or via the two bridging hydrogen atoms) rather than 'through space' (i.e. in a direct manner). Interestingly, based on IQA-defined interatomic exchange-correlation energies ( $\left.V_{\mathrm{XC}}^{\mathrm{A}, \mathrm{B}}\right)$, Pendás et $a l^{[57]}$ have previously shown that an AIL between atoms $\mathrm{H} 7$ and $\mathrm{H} 8$ is more likely to form than between atoms B1 and B4, but less likely than the existing AIL-linked H-atoms. Their results can easily be rationalized with the isosurfaces in Figure 7 (c) and (d); they show that the manner through which electrons are delocalized contributes cooperatively to the $\mathrm{H}$ atoms linked by an AIL as well as the AIL-free interaction between $\mathrm{H} 7$ and $\mathrm{H} 8$. 
All the above analyses demonstrate that atom-pairs which made major contributions at $\mathrm{BCP}(\mathrm{B} 1, \mathrm{H} 7)$ delocalise a substantial quantity of their ED in regions other than directly between each other, highlighting the large multicentre character of most AILs in diborane.

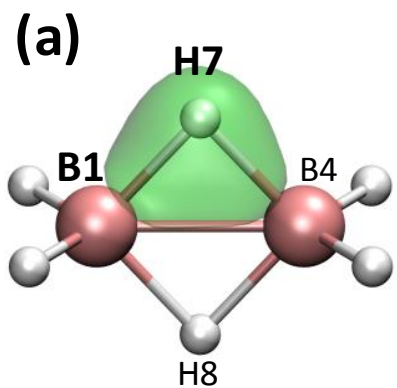
$\mathcal{D}_{\mathrm{B} 1, \mathrm{H}}(\mathrm{BCP})=0.01641$ a.u.
Isovalue $=0.010$ a.u.

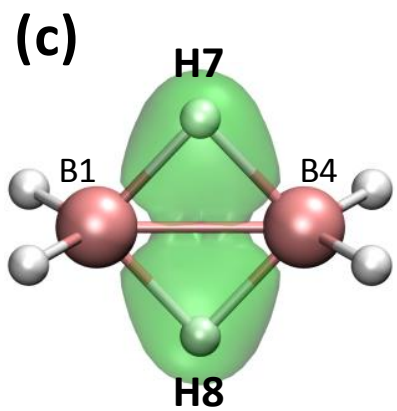

$\mathcal{D}_{\mathrm{H} 7, \mathrm{H} 8}(\mathrm{BCP})=0.00463$ a.u. Isovalue $=0.004$ a.u.

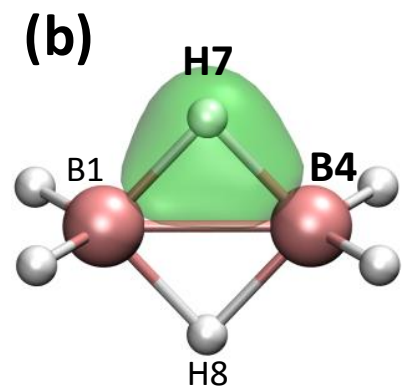

$\mathcal{D}_{\mathrm{B} 4, \mathrm{H} 7}(\mathrm{BCP})=0.01531$ a.u. Isovalue $=0.010$ a.u.

(d)

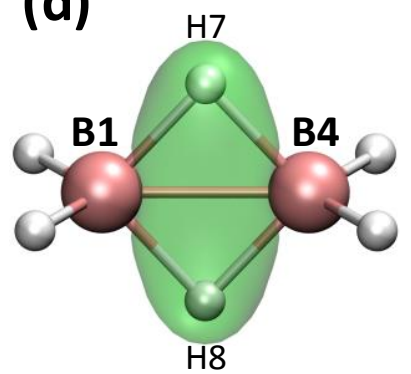

$\mathcal{D}_{\mathrm{B} 1, \mathrm{~B} 4}(\mathrm{BCP})=0.00300$ a.u. Isovalue $=0.002$ a.u.

Figure 7. Deloc-ED 3D-isosurfaces of the major constructive electron correlation contributing atompairs with respect to $\mathrm{BCP}(\mathrm{B} 1, \mathrm{H} 7)$ : (a) $\mathcal{D}_{\mathrm{B} 1, \mathrm{H} 7}(33 \%)$, (b) $\mathcal{D}_{\mathrm{B} 4, \mathrm{H} 7}(31 \%)$, (c) $\mathcal{D}_{\mathrm{H} 7, \mathrm{H} 8}(9 \%)$ and (d) $\mathcal{D}_{\mathrm{B} 1, \mathrm{~B} 4}(6 \%)$.

\subsection{Typical carbon-carbon covalent bonding interaction in 'linear' $n$-butane}

Figures $8(\mathbf{a}$ and $\mathbf{b})$ show the FALDI-based decomposition of the constructive electron correlation in 'linear' $n$-butane (with respect to $\mathrm{BCP}(\mathrm{C} 9, \mathrm{C} 12)$ ). Additional FALDI decompositions are included in PART 2 of the Supplementary Information. Unsurprisingly, atom-pair $\mathrm{C} 9, \mathrm{C} 12$ contributes by far the greatest amount of deloc-ED of a bonding nature, accounting for $84 \%$ of the total. This result is in agreement with the general interpretation of a covalent 'single' bond as being a two-centre interaction. A 3D-isosurface of atom-pair C9,C12 deloc-ED distribution is shown in Figure 9(a); it reveals a clear XC-channel generally confined within the $\mathrm{C} 9, \mathrm{C} 12$ interatomic region. The $\mathrm{C} 9, \mathrm{C} 12$ deloc-ED distribution is therefore qualitatively very different to that of the atom-pair B1,H7 in diborane (Figure $7(\mathbf{a})$ ). Interestingly, our FALDI analyses reveal that this carbon-carbon interaction is not 
entirely bicentric. Note that $16 \%$ of the total deloc-ED resulting from constructive electron correlation at $\mathrm{BCP}(\mathrm{C} 9, \mathrm{C} 12)$ is due to other (secondary) atom-pairs. These secondary contributions come mostly from (i) the sum of four equivalent 'carbon atom-hydrogen atom' atom-pairs, abbreviated as C--H (i.e. $\mathrm{C} 9-\{\mathrm{H} 13, \mathrm{H} 14\}$ plus $\mathrm{C} 12-\{\mathrm{H} 10, \mathrm{H} 11\})$, accounting for $7 \%$ and (ii) the four 'carbon atom-carbon atom' atom-pairs (i.e. $\mathrm{C} 2-\{\mathrm{C} 9, \mathrm{C} 12\}$ plus $\mathrm{C} 3-$ $\{\mathrm{C} 12, \mathrm{C} 9\})$ accounting for $6 \%$ of the total. 3D-isosurfaces of these contributions,
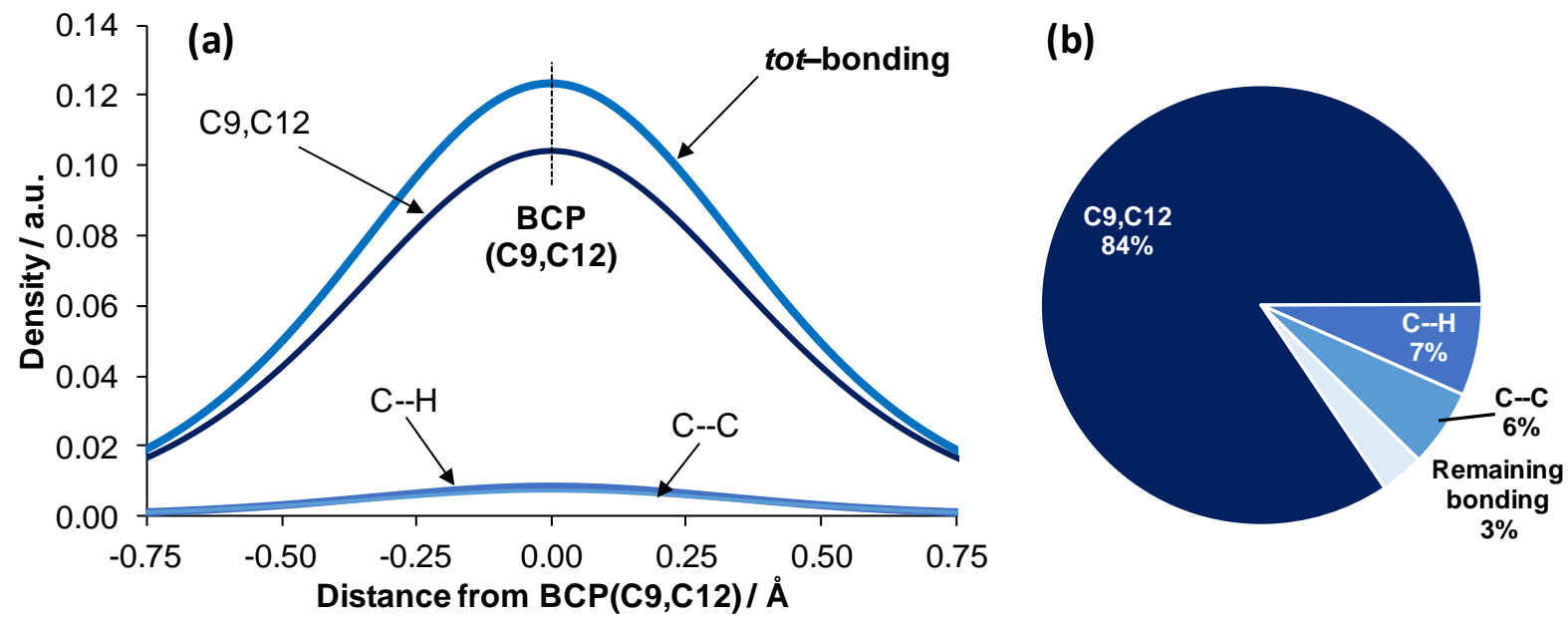

Figure 8. (a) FALDI-based decomposition of the constructive electron correlation (i.e. deloc-ED of a bonding nature) along the indicated vector for $\mathbf{3}$ in Figure 1, in 'linear' $n$-butane, into the major contributing atom-pairs. (b) Pie-chart summarizing percentage-wise the major contributing factors at the $\mathrm{BCP}(\mathrm{C} 9, \mathrm{C} 12)$.

(a)

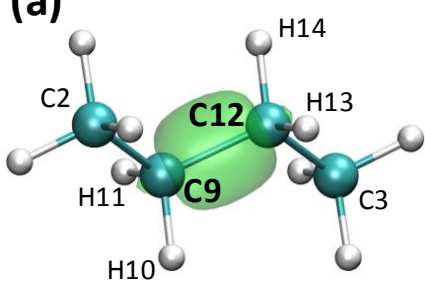

$\mathcal{D}_{\mathrm{cg}, \mathrm{C} 12}(\mathrm{BCP})=\mathbf{0 . 1 0 4 1 3}$ a.u. Isovalue $=0.020$ a.u. (b)

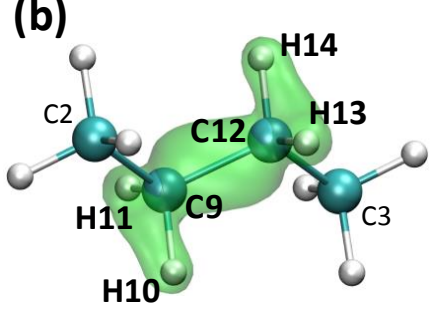

$\mathcal{D}_{\text {C-H }}(B C P)=0.00829$ a.u. Isovalue $=0.002$ a.u.

(c)

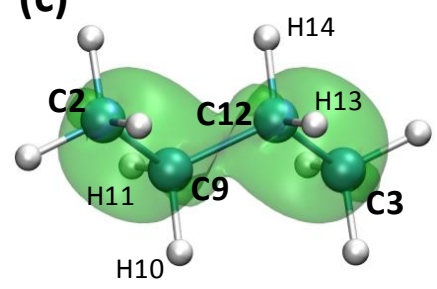

$\mathcal{D}_{\text {C-C }}(B C P)=0.00704$ a.u

Isovalue $=0.007$ a.u.

Figure 9. Deloc-ED 3D-isosurfaces of the major constructive electron correlation contributions with respect to $\mathrm{BCP}(\mathrm{C} 9, \mathrm{C} 12)$ in 'linear' $n$-butane: (a) $\mathcal{D}_{\mathrm{C} 9, \mathrm{C} 12}(84 \%)$, (b) $\mathcal{D}_{\mathrm{C}-\mathrm{H}}(7 \%)$ and $(\mathbf{c}) \mathcal{D}_{\mathrm{C}-\mathrm{C}}(6 \%)$. 
Figures 9(b and c), reveal how atom-pairs of these 'distant' interactions also concentrate ED along $\operatorname{AIL}(\mathrm{C} 9, \mathrm{C} 12)$. In this context, the $\mathrm{C} 9-\mathrm{C} 12$ covalently bonded interaction, as measured at its associated BCP, can only be approximated as two-centre.

\subsection{Comparison of two different $\mathrm{M}-\mathrm{C}$ bonding interactions in carbene complexes}

The differences between $\mathrm{M}-\mathrm{C}$ bonding interactions in Schrock, Fischer and several other types of carbene complexes have been a constant focal point of inorganic chemistry research for a number of decades. ${ }^{[21]}$ It is generally accepted that heteroatoms bonded directly to the carbene carbon atom in Fischer carbene complexes (such as an oxygen, 5 in Figure 1) enhance $\pi$-backbonding from the metal atom, as well as $\sigma$-donation from the ligand. From a QTAIM, i.e. tot-ED topology perspective, however, the $\mathrm{M}-\mathrm{C}$ bonds of the Schrock and Fischer carbene complexes appear to be almost identical, as both consist of (i) an AIL connecting the metal and carbon atoms, (ii) a positive Laplacian value at $\mathrm{BCP}$, (iii) a $\mid \mathrm{V} / \mathrm{G}$ ratio larger than one and (iv) a DI in the range of $1.0<\mathrm{DI}(\mathrm{M}, \mathrm{C})<1.5$ (shown in PART 3 of the Supplementary Information). It was then of interest and importance to find out whether the approach presented in this work can quantify the degree and point correctly at the reason for the difference of multicentre bonding characters of the two M-C AILs in the Schrock and Fischer (4 and 5, respectively, in Figure 1) carbene complexes.

Figures 10(a and c) show the FALDI-based decomposition of concentrated deloc-ED in 4 and 5 with respect to the relevant $\mathrm{M}-\mathrm{C}$ BCPs. Additional FALDI decompositions are included in PART 2 of the Supporting Information. Figures 10(a and c) show that the general shapes of the trends pertaining to structures $\mathbf{4}$ and $\mathbf{5}$ are similar as they only differ in relative magnitude. The primary $\mathrm{M}-\mathrm{C}$ interaction in $\mathbf{4}$ accounts for comparatively more (80\%) of the total deloc-ED of a bonding nature as compared to that in $\mathbf{5}(63 \%)$.

Clearly, the AIL linking the $\mathrm{M}$ and carbene $\mathrm{C}$ atoms in the Schrock complex is considerably more bicentric than in the Fischer complex. Importantly, the heteroatom O13 is indeed mainly responsible for the comparatively larger degree of multicentre bonding character in 5 (Figure 10(b)), in accord with common knowledge. Two atom-pairs containing atom $\mathrm{O} 13$, that with the carbene carbon atom $\left(\rho_{\mathrm{BCP}}=0.00309\right.$ a.u. $)$ and with the carbene metal atom $\left(\rho_{\mathrm{BCP}}=0.00096\right.$ a.u.) account for a significant $12 \%$ of the total constructive electron correlation at $\mathrm{BCP}(\mathrm{Cr} 1, \mathrm{C} 12)$. In comparison, atom-pair combinations of the nonheteroatoms $\mathrm{H} 23$ or $\mathrm{H} 24$ (equivalent due to symmetry of the molecular structure) in 4, with the carbene carbon atom ( $\rho=0.00200$ a.u.) and with the carbene metal atom $(\rho=0.00134$ 
a.u.) account for a much smaller $7 \%$ of the total deloc-ED of a bonding nature at $\mathrm{BCP}(\mathrm{Ti1}, \mathrm{C} 22)$.

3D-isosurfaces of the major contributions to the deloc-ED of a bonding nature in both carbenes are comparable, showing similar features as found in the $n$-butane; they are included in PART 4 of the Supporting Information together with short discussion.
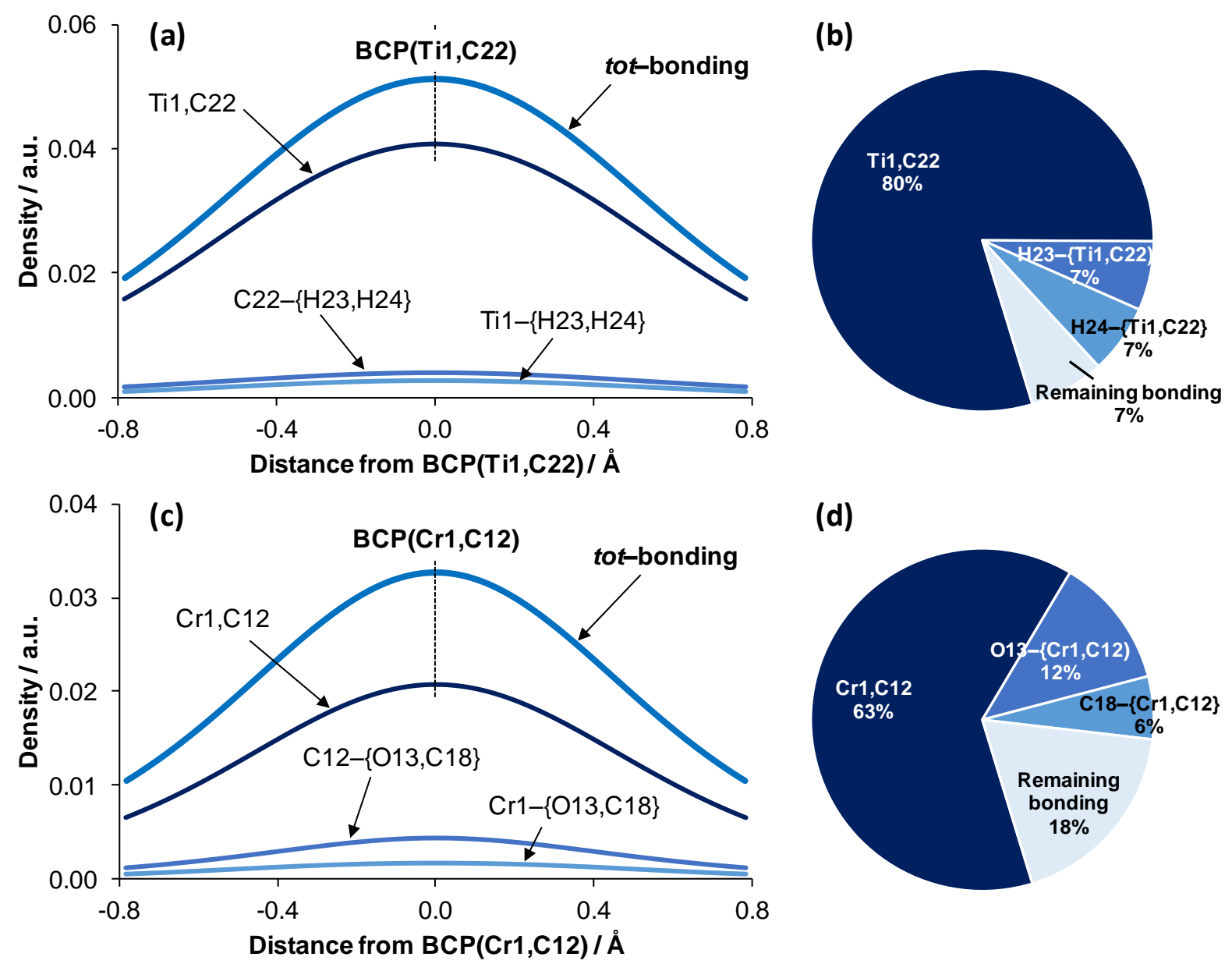

(d)

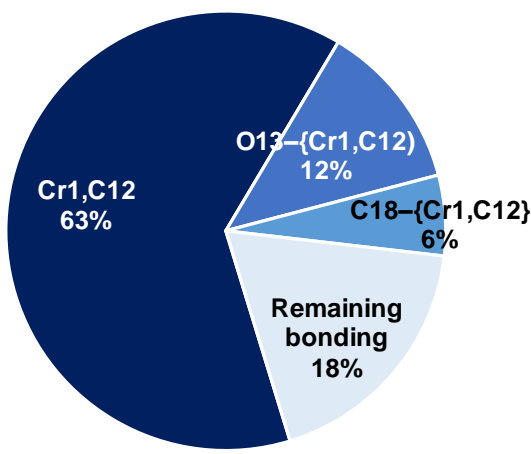

Figure 10. (a) Comparison of the FALDI-based decompositions of the constructive electron correlation (i.e. deloc-ED of a bonding nature) with respect to the M-C BCPs in the Schrock and Fischer carbene complexes, into the major contributions made by the indicated atom-pairs. (b) Piecharts summarising percentage-wise the major contributions at the M-C BCPs in $\mathbf{4}$ and $\mathbf{5}$.

\subsection{Comparative analysis}

A comparison of the relative contributions made to the total deloc-ED of a bonding nature by the primary, largest secondary and sum of remaining interactions at BCPs associated with the five investigated bonding interactions is shown in Figure 11. The most striking observation one can make is the fact that 'pure' (i.e. 100\%) bi-centre interactions might not exist at all, 
except in an isolated diatomic molecules; note a significant $(16 \%)$ contribution made by atom-pairs other than the C9-C12 interaction (a classical single covalent bond) in $n$-butane.

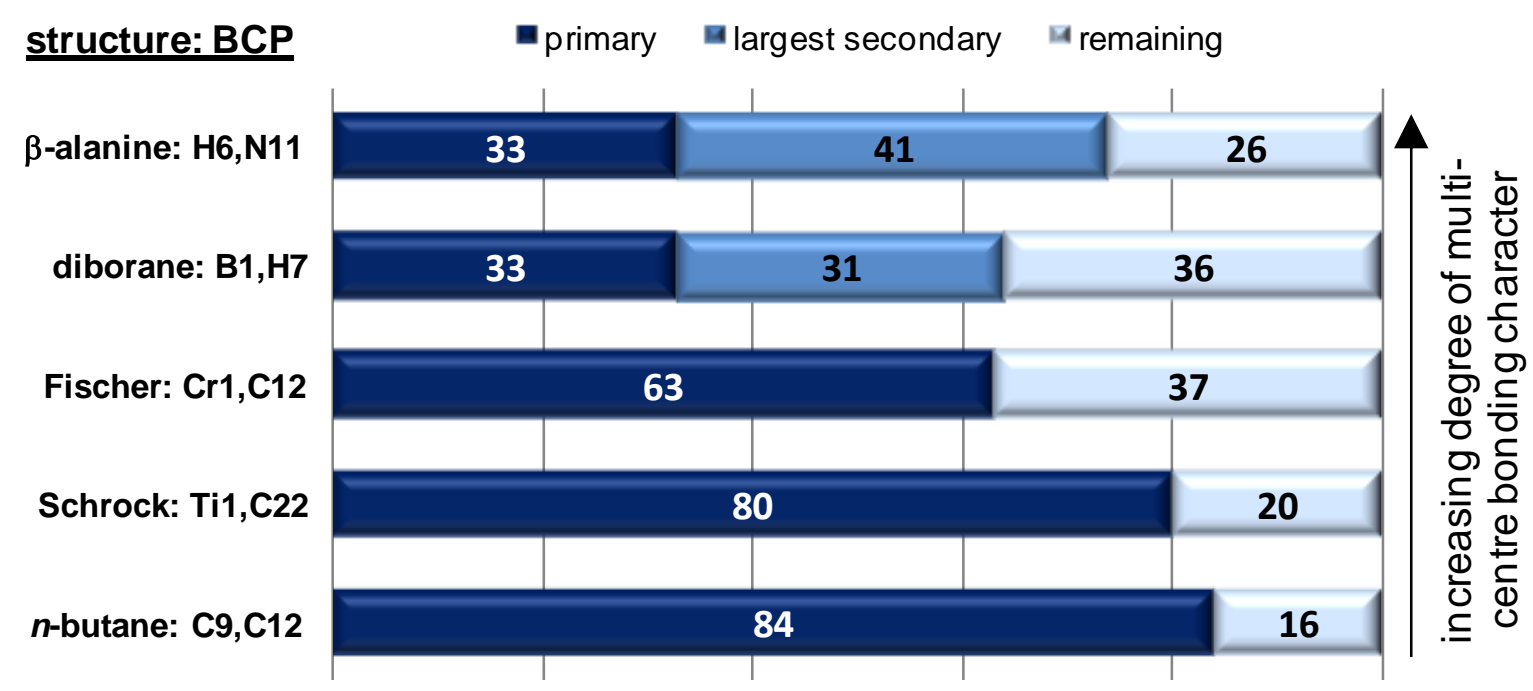

percentage contribution

Figure 11. Comparison of the relative degree of multicentre bonding character of the five bonding interactions investigated in this study. Values of specific atom-pair deloc-ED contributions to the total bonding deloc-ED at a relevant $\mathrm{BCP}$ are expressed as percentages. Primary contributions indicate the deloc-ED associated with an indicated atom-pair that is connected by an AIL.

The second surprising observation is the comparable degree of multicentre character discovered for the well-known case of diborane (as discussed in detail for atom-pair B1,H7) and classical intramolecular H-bonding interaction in $\beta$-alanine. In both cases, the primary interactions (atom-pairs H6,H11 and B1,H7 in $\beta$-alanine and diborane, respectively) account for just $33 \%$ of the total deloc-ED of bonding nature. It is important to stress that in the gas phase the largest secondary interaction (the atom pair $\mathrm{O} 5, \mathrm{H} 6$, or the $\mathrm{X}-\mathrm{H}$ bond) contributed more $(41 \%)$ to the concentrated deloc-ED at $\mathrm{BCP}(\mathrm{H} 6, \mathrm{~N} 11)$ in $\beta$-alanine than the primary interaction (Fig. 4). However, the reverse trend holds in the aqueous phase. Considering diborane's most significant secondary contribution to the bonding deloc-ED at $\mathrm{BCP}(\mathrm{B} 1, \mathrm{H} 7)$, it amounts to $31 \%$, which is nearly the same as found for the primary interaction. This nicely exemplifies the predominant tricentric character of a B,H AIL.

Finally, relative to the Schrock carbene, a larger degree of multicentre character of the M$\mathrm{C}$ bonding interaction was fully confirmed in the Fischer carbene (atom-pair Cr1,C12). Possibly surprisingly, we have discovered that the degree of bicentre $\mathrm{M}-\mathrm{C}$ bonding interaction in the Schrock carbene is comparable to that found for the classical single covalent bond in $n$-butane; we found that the primary interactions, Ti1,C22 and $\mathrm{C} 9, \mathrm{C} 12$, 
contributed 80 and $84 \%$, respectively, to the total concentrated deloc-ED at the respective BCPs.

All these results show how the presence of an AIL, connecting only two nuclei, can be misleading in multi-atomic molecules. In fact, our results reveal that AILs are surprisingly holistic in nature, despite their simple connectivity within a molecular graph. Figure 11 clearly illustrates that there are many atom-pairs that contribute significantly to the total deloc-ED in a constructive (concentrating) manner, hence facilitating the emergence of the AIL. This clearly demonstrates that care should be taken whenever an AIL is used to indicate and interpret the interaction between just two atoms. This comment applies, of course, to any method based on ED concentration, including NCI.

\section{Conclusions}

The concept of two-centre interatomic interactions is, for the most part, a chemist's simplification within a molecular collection of nuclei and electrons interacting holistically and simultaneously. The AIL, which is also a physical observable, is usually interpreted as the result of a two-centre interaction and is commonly followed by an analysis of topological properties at a specific BCP to describe a di-atomic interaction. While many multicentre indices have been reported in the literature, which can recover the multicentre nature of interatomic interactions, none of these indices address the real-space AIL itself.

Our FALDI density decomposition scheme, which measures the ED contributions of atoms and atom-pairs throughout all molecular space, provides the tools that are necessary to understand AILs on a holistic level. We have extended FALDI in this work by decomposing one of the fundamental ED properties of interatomic interactions - the concentration, depletion or reduction of ED - into contributions from all atomic pairs within the molecule. Such an approach allows a quantification and visualization of how the atoms of a primary interaction (atoms linked by an AIL) contribute to ED relative to all other atom-pairs, thereby arriving at a multicentre interpretation of an AIL. We have investigated five simple interactions linked by an AIL, ranging from formal two-centre to formal three-centre as well as interactions with partial multicentre character. Our results are fully consistent with general interpretations of multicentre bonding, despite focussing on the multicentre character of the AIL as measured in the vicinity of a BCP. We also note that our approach is not limited to atoms linked by an AIL, and can be used in conjunction with other QCT techniques, such as NCI. 
AILs have previously been interpreted as 'privileged exchange channels' by Pendás et $a l .{ }^{[55]}$ This interpretation has been tested by Tognetti and Joubert ${ }^{[53,54]}$ by comparing the QTAIM-defined DI or the IQA-defined interatomic XC energy of the primary interaction (linked by an AIL) with the largest competing secondary interaction. While they could show that the primary interaction is indeed privileged (i.e. larger DI or XC energy than competing interactions) for strong interactions, they identified a number of cases where their approach did not give consistent results. We have shown that, unlike the total integrated DI (as defined in QTAIM), real-space distributions of FALDI-defined deloc-ED can be negative in certain regions of space. The sign of deloc-ED at any given coordinate, as well as the sign of its partial second derivative, is a result of constructive, nonconstructive or deconstructive simultaneous overlap of MOs across two atomic basins. Some of these atom-pairs' delocalized ED concentrates ED in the same interatomic region, thereby facilitating the formation of an AIL, whereas some atom-pairs' delocalized ED depletes or reduces ED, thereby hindering and competing against the formation of an AIL. This observation led to our labels of bonding, nonbonding or antibonding of any specific atom-pair's deloc-ED in a given region of space in analogy to MO bonding theory. Therefore, any AIL, hence also a 'privileged exchange channel', is not simply a bridge of ED linking two atoms but rather the result of multiple bonding contributions that compensate over the competing nonbonding and antibonding contributions. This can be seen as a new interpretation of a "privileged exchange channel' that does not impose largest contribution to be made by the atom-pair linked by an AIL. As a matter of fact, the intramolecular H-bonding in $\beta$-alanine fully supports this, as the primary interaction (linked by an AIL) contributed significantly less when compared with a secondary one. Furthermore, because our approach also has the capability for the visualisation and quantification of 'privileged exchange channels' (e.g. Figure 9) we noted, by inspecting deloc-ED isosurfaces, that most of the secondary bonding contributions investigated in this work occur through the primary interaction, i.e. along the AIL, as opposed to forming direct channels through space.

Bader et al. have previously stated ${ }^{[49]}$ that QTAIM and MO theories are fully consistent, with the primary link between the two being the DI. We note, however, that only through the visualisation in real-space of FALDI's deloc-ED distributions can the information inherent in MO theory be fully expanded within the realms of QTAIM. For instance, DIs alone do not recover the manner in which atom-pair $\mathrm{O} 5, \mathrm{H} 6$ concentrates deloc-ED at $\mathrm{BCP}(\mathrm{H} 5, \mathrm{~N} 11)$ in $\beta$ alanine, nor the manner in which electrons shared between atoms B1 and $\mathrm{H} 7$ in diborane are 
delocalized almost equally across the entire $\mathrm{B} \cdots \mathrm{H} \cdots \mathrm{B}$ region. In addition, DI values are always positive whereas, as shown in Figure 3(c), due to deconstructive electron correlation, the electrons shared between two atoms can reduce the tot-ED, leading to a different energetic consequence for an interaction. Importantly, the decomposition of the total deloc$\mathrm{ED}$ in the vicinity of a $\mathrm{BCP}$ in terms of bonding, nonbonding and antibonding is consistent with both Bader's as well as MO theories.

The ability for chemists to understand and quantify the holistic nature of AILs should help alleviate conceptual challenges within QCT, especially for controversial AILs, since the properties of the ED at a BCP are commonly used in the classification and evaluation of interatomic interactions. Our assertion that the manner in which electrons are delocalized between atoms (deloc-ED distributions) is as important as the total amount of delocalized electrons (DI), should also aid the distillation of the wealth of information within MOs and the wavefunction into an atom-centric, density-based view of chemical bonding.

\section{Conflicts of interest}

There are no conflicts of interest to declare.

\section{Acknowledgements}

This work is based on the research supported in part by the National Research Foundation of South Africa (Grant Number 105855). The authors gratefully acknowledge the Centre for High Performance Computing (CHPC), South Africa, for providing computational resources to this research project.

\section{References}

[1] B. Silvi, J. Mol. Struct. 2002, 614, 3-10.

[2] W. C. Price, J. Chem. Phys. 1947, 15, 614.

[3] J. S. Kasper, C. M. Lucht, D. Harker, Acta Cryst. 1950, 3, 436-455.

[4] K. Hedberg, M. E. Jones, V. Shomaker, J. Am. Chem. Soc. 1951, 73, 3538-3539.

[5] C. E. Nordman, W. N. Lipscomb, J. Chem. Phys. 1953, 21, 1856-1864.

[6] L. Lavine, W. N. Lipscomb, J. Chem. Phys. 1954, 22, 614-620.

[7] M. Elian, R. Hoffman, Inorg. Chem. 1975, 14, 1058-1076.

[8] R. H. Summerville, R. Hoffman, J. Am. Chem. Soc. 1979, 101, 3821-3831.

[9] R. Ponec, G. Lendvay, J. Chaves, J. Comput. Chem. 2008, 29, 1387-1398. 
[10] N. M. Kostic. R. F. Fenske, Inorg. Chem. 1983, 22, 666-671.

[11] R. Bau, R. G. Teller, S. W. Kirtley, T. F. Koetzle, Acc. Chem. Res. 1979, 12, 176-183.

[12] F. Mota, J. S. Miller, J. J. Novoa, J. Am. Chem. Soc. 2009, 131, 7699-7707.

[13] I. Garcia-Yoldi, J. S. Miller, J. J. Novoa, J. Phys. Chem. A 2009, 113, 7124-7132.

[14] W. Wang, Y. Kan, L. Wang, S. Sun, Y. Qiu, J. Phys. Chem. C 2014, 118, 28746-28756.

[15] J. S. Miller, J. J. Novoa, Acc. Chem. Res. 2007, 40, 189-196.

[16] B. Silvi, C. Gatti, J. Phys. Chem. A 2000, 104, 947-953.

[17] E. Arunan, G. R. Desiraju, R. A. Klein, J. Sadlej, S. Scheiner, I. Alkorta, D. C. Clary, R. H. Crabtree, J. J. Dannenberg, P. Hobza, H. G. Kjaergaard, A. C. Legon, B. Mennucci, D. J. Nesbitt, Pure Appl. Chem. 2011, 83, 1619-1636.

[18] E. Arunan, G. R. Desiraju, R. A. Klein, J. Sadlej, S. Scheiner, I. Alkorta, D. C. Clary, R. H. Crabtree, J. J. Dannenberg, P. Hobza, H. G. Kjaergaard, A. C. Legon, B. Mennucci, D. J. Nesbitt, Pure Appl. Chem. 2011, 83, 1637-1641.

[19] I. Cukrowski, D. M. E. van Niekerk, J. H. de Lange, Struct. Chem. 2017, 5, 1429-1444.

[20] I. Cukrowski, J. H. de Lange, A. S. Adeyinka, P. Mangondo, Comp. Theo. Chem. 2015, 1053, 60-76.

[21] D. Bourissou, O. Guerret, F. P. Gabbaï, G. Bertrand, Chem. Rev. 2000, 100, 39-92.

[22] R. F. W. Bader in Atoms in Molecules: A Quantum Theory, Oxford University Press, Oxford, 1990.

[23] P. Dem'yanov, P. Polestshuk, Chem.-Eur. J. 2012, 18, 4982-4993.

[24] M. Mandado, A. M. Graña, R. A. Mosquera, Phys. Chem. Chem. Phys. 2004, 6, 43914396.

[25] L. J. Farrugia, H. M. Senn, J. Phys. Chem. A 2011, 116, 738-746.

[26] C. F. Matta, J. Hernández-Trujillo, T. H. Tang, R. F. W. Bader, Chem.-Eur. J. 2003, 9, 1940-1951.

[27] P. Bultinck, M. Rafat, R. Ponec, B. Van Gheluwe, R. Carbo-Dorca, P. Popelier, J. Phys. Chem. A 2006, 110, 7642-7648.

[28] R. Ponec, I. Mayer, J. Phys. Chem. A 1997, 101, 1738-1741.

[29] R. Ponec, G. Yuzhakov, D. L. Cooper, Theor. Chim. Acta 2004, 5, 419-430.

[30] R. F. W. Bader, C. Gatti, Chem. Phys. Lett. 1998, 287, 233-238.

[31] C. Gatti, F. Cargnoni, L. Bertini, J. Comp. Chem. 2003, 24, 422-436.

[32] E. Monza, C. Gatti, L. L. Presti, E. Ortoleva, J. Phys. Chem. A 2011, 115, 12864-12878.

[33] J. H. de Lange, I. Cukrowski, J. Comp. Chem. 2017, 38, 981-997. 
[34] E. R. Johnson, S. Keinan, P. Mori-Sanchez, J. Contreras-Garcia, A. J. Cohen, W. Yang, J. Am. Chem. Soc. 2010, 132, 6498-6506.

[35] M. J. Frisch, G. W. Trucks, H. B. Schlegel, G. E. Scuseria, M. A. Robb, J. R. Cheeseman, G. Scalmani, V. Barone, B. Mennucci, G. A. Petersson, H. Nakatsuji, M. Caricato, X. Li, H. P. Hratchian, A. F. Izmaylov, J. Bloino, G. Zheng, J. L. Sonnenberg, M. Hada, M. Ehara, K. Toyota, R. Fukuda, J. Hasegawa, M. Ishida, T. Nakajima, Y. Honda, O. Kitao, H. Nakai, T. Vreven, J. A., Jr. Montgomery, J. E. Peralta, F. Ogliaro, M. Bearpark, J. J. Heyd, E. Brothers, K. N. Kudin, V. N. Staroverov, R. Kobayashi, J. Normand, K. Raghavachari, A. Rendell, J. C. Burant, S. S. Iyengar, J. Tomasi, M. Cossi, N. Rega, J. M. Millam, M. Klene, J. E. Knox, J. B. Cross, V. Bakken, C. Adamo, J. Jaramillo, R. Gomperts, R. E. Stratmann, O. Yazyev, A. J. Austin, R. Cammi, C. Pomelli, J. W. Ochterski, R. L. Martin, K. Morokuma, V. G. Zakrzewski, G. A. Voth, P. Salvador, J. J. Dannenberg, S. Dapprich, A. D. Daniels, Ö. Farkas, J. B. Foresman, J. V. Ortiz, J. Cioslowski, D. J. Fox, Gaussian 09, Revision D.01; Gaussian, Inc.: Wallingford CT, 2009.

[36] S. Grimme, Wiley Interdiscip. Rev. Comput. Mol. Sci. 2011, 1, 211-228.

[37] T. A. Keith, AIMAll (Version 16.10.31); TK Gristmill Software: Overland Park KS, USA, 2016. Available at: aim.tkgristmill.com.

[38] W. Humphrey, A. Dalke, K. Schulten, J. Molec. Graphics 1996, 14, 33-38.

[39] A. M. K. Müller, Phys. Lett. A 1984, 105, 446-452.

[40] D. L. Cooper, R. Ponec, Phys. Chem. Chem. Phys. 2008, 10, 1319-1329.

[41] M. Bühl, H. Kabrede, J. Chem. Theory Comput. 2006, 2, 1282-1290.

[42] P. Bultinck, D. L. Cooper, R. Ponec, J. Phys. Chem. A 2010, 114, 8754-8763.

[43] R. Ponec, J. Math. Chem. 1997, 12, 323-333.

[44] R. Ponec, J. Math. Chem. 1998, 23, 85-103.

[45] R. Ponec, D. L. Cooper, Faraday Discuss. 2007, 135, 31-42.

[46] E. Francisco, A. M. Pendás, A. Costales, Phys. Chem. Chem. Phys. 2014, 16, 45864597.

[47] R. F. W. Bader, M. E. Stephens, J. Am. Chem. Soc. 1975, 97, 7391-7399.

[48] X. Fradera, M. A. Austen, R. F. W. Bader, J. Phys. Chem. A 1999, 103, 304-314.

[49] F. Cortés-Guzmán, R. F. W. Bader, Coord. Chem. Rev. 2005, 249, 633-662.

[50] I. Sumar, R. Cook, P. W. Ayers, C. F. Matta, Comp. Theor. Chem. 2015, 1070, 55-67.

[51] I. Sumar, R. Cook, P. W. Ayers, C. F. Matta, Phys. Scr. 2016, 91, 013001-0130014. 
[52] M. J. Timm, C. F. Matta, L. Massa, L. Huang, J. Phys. Chem. A 2014, 118, 1130411316.

[53] V. Tognetti, L. Joubert, J. Chem. Phys. 2013, 138, 024102-024109.

[54] O. A. Syzgantseva, V. Tognetti, L. Joubert, J. Phys. Chem. A 2013, 117, 8969-8980.

[55] A. M. Pendás, E. Francisco, M. A. Blanco, C. Gatti, Chem.-Eur. J. 2007, 13, 93629371.

[56] R. Ponec, F. Uhlik, J. Mol. Struct. (Theochem) 1997, 391, 159-168.

[57] M. García-Revilla, E. Francisco, P. L. A. Popelier, A. M. Pendás, Chem. Phys. Chem. 2013, 14, 1211-1218. 


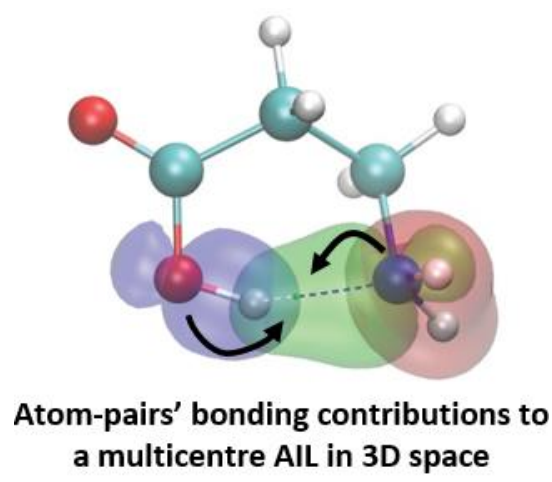

3D decomposition of an atomic interaction line (AIL) into contributions made by multiple atompairs to the total delocalized electron density at a BCP reveals the holistic nature of Bader's bond path linking only two atoms. A novel method, applied on five classical bonding interactions, reveals main atom-pairs' bonding, nonbonding or antibonding contributions to and various degrees of multicentre character of these interactions. 